\title{
Experimental investigation of the transonic buffet cycle on a supercritical airfoil
}

\author{
A. D'Aguanno ${ }^{1}$ (I) F. F. J. Schrijer ${ }^{1}$ • B. W. van Oudheusden ${ }^{1}$
}

Received: 15 April 2021 / Revised: 1 September 2021 / Accepted: 6 September 2021 / Published online: 1 October 2021

(c) The Author(s) 2021

\begin{abstract}
Transonic buffet behaviour of the supercritical airfoil OAT15A was investigated experimentally at flow conditions $M a=0.7$ and $\alpha=3.5^{\circ}$, using schlieren and particle image velocimetry (PIV). The general behaviour of the buffet cycle was characterised with short-exposure schlieren visualisation and phase-averaged PIV measurements. A spectral analysis showed that the shock oscillation occurs with a dominant contribution at $160 \mathrm{~Hz}$ ( $\mathrm{St}=0.07$, in good agreement with the literature) and between 25 and $55 \%$ of the chord of the airfoil. Proper Orthogonal Decomposition (POD) was applied to the PIV data to extract the main modes connected with buffet. It is found that the first three most energetic modes capture around $65 \%$ of the total fluctuating kinetic energy. The first and the third modes have a main frequency peak at $160 \mathrm{~Hz}$ and are well representing the separated area and the shock oscillation. The second mode was, instead, associated with an asymmetrical behaviour of the separated area and of the shear layer and displays a main peak at $320 \mathrm{~Hz}$, being double the main buffet cycle frequency. Finally, it was shown that by using the 11 most energetic POD modes, an accurate reduced-order model (ROM) is obtained, which when subtracted from the instantaneous velocity fields allows the visualisation of the small-scale structures present in the flow, such as the upstream travelling waves (UTWs) and the vortex shedding in the separated area near the trailing edge. The analysis allowed to estimate the velocity of the UTWs, obtaining values in good agreement with the literature. In contrast, the analysis of the vortex dynamics in the trailing edge area revealed that vortices shed at the shock foot, which convect downstream in an area detached from the airfoil surface, cannot be considered responsible for the creation of UTWs in view of the mismatch in frequency of the two phenomena.
\end{abstract}

\footnotetext{
A. D'Aguanno

A.Daguanno@tudelft.nl

1 Delft University of Technology, Faculty of Aerospace

Engineering, Aerodynamics Department, Kluyverweg 2,

2629HS Delft, The Netherlands
} 


\section{Graphic abstract}
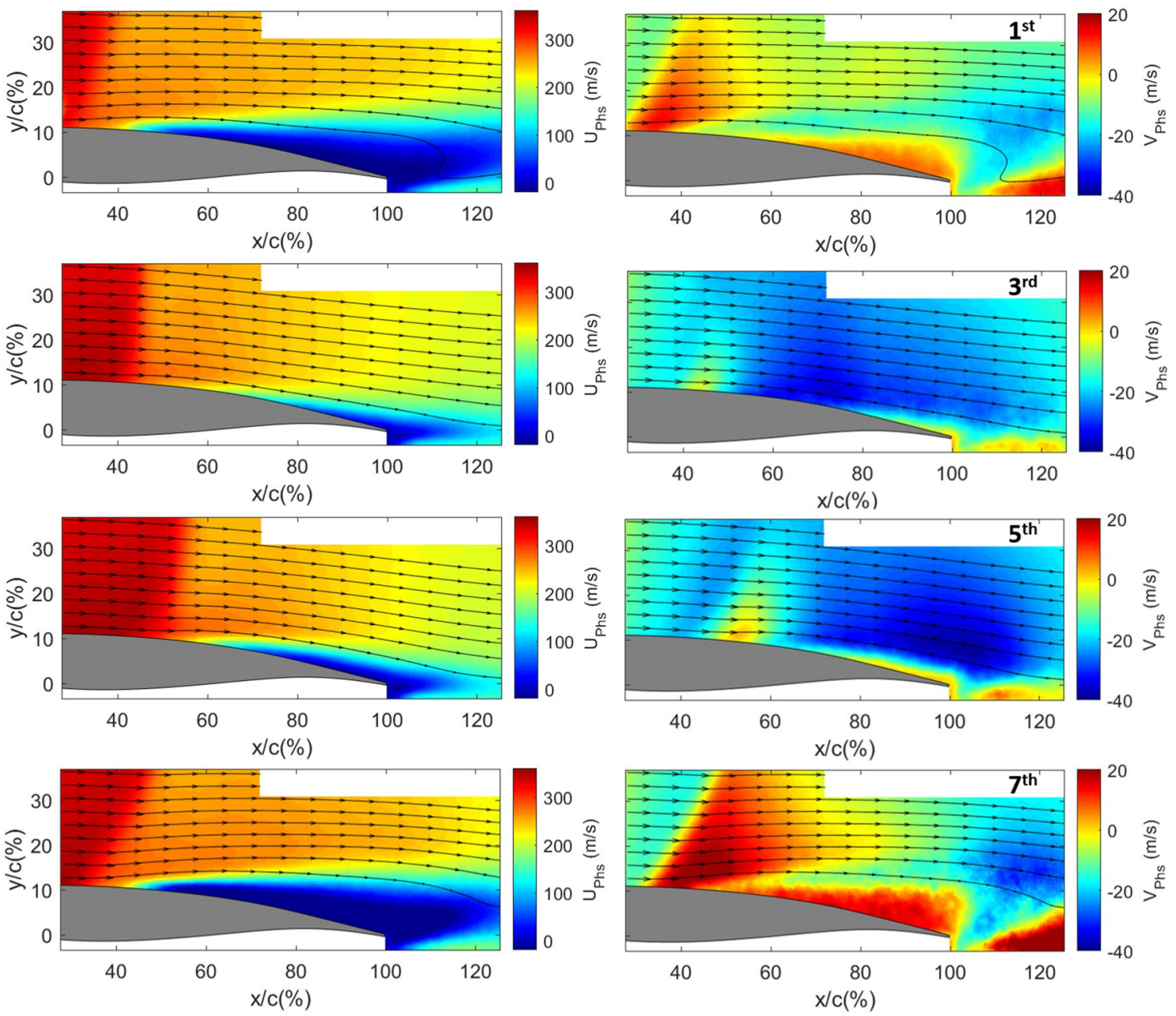

\section{Introduction}

Transonic buffet consists of an oscillation of the shockwave (SW) on the suction side of a wing/airfoil (for Type II buffet, which is object of this study) that may occur for a certain range of $M a, \alpha$ and $R e$ values. For 2D profiles, this results in a periodic variation in the aerodynamic loads, while the interaction with the structure could eventually result in its failure due to fatigue. For these reasons, it is of paramount importance that an airplane flies in a region of the flight envelope in which such a phenomenon does not occur. In order to ensure safe operation, the aircraft regulation authorities impose conservative margins on the flight envelope. Therefore, to optimise the performance of a civil aircraft, several studies on the understanding and on the possibility of controlling transonic buffet have been performed, see, for example, the review by Giannelis et al. (2017).
Early investigations of transonic buffet were undertaken by Hilton and Fowler (1952), and a first attempt to explain the transonic buffet mechanism was made by Lee (1990), who described the shock buffet oscillation as being sustained by a feedback mechanism. In this model, disturbances

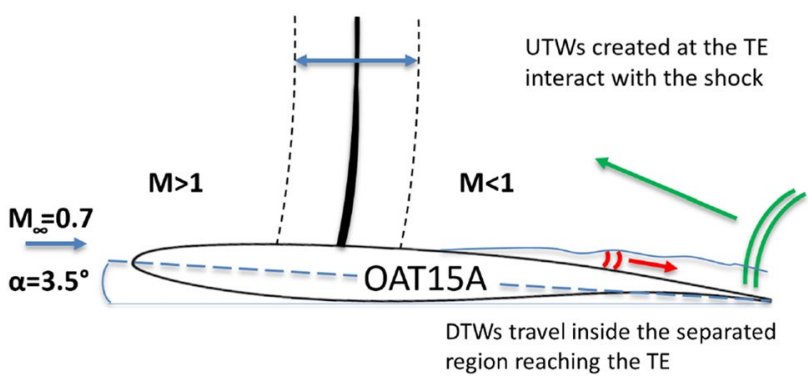

Fig. 1 Sketch of transonic buffet feedback mechanism 
created at the shock foot travel downstream towards the trailing edge inside the separated area (indicated in red in Fig. 1). Once these downstream travelling waves (DTWs) reach the trailing edge, upstream travelling waves (UTWs) are created in order to respect the Kutta condition. These UTWs (indicated in green) travel upstream towards the shockwave thereby sustaining the shock oscillation. Although this model gave results that are reasonably close to the ones reported in the literature, it was found not accurate enough to compute the buffet frequency, as shown by Deck (2005) and Jacquin et al. (2009).

The model introduced by Lee was updated by Deck (2005), who considered the UTWs to be able to travel not only along the suction side, but along the pressure side as well, which resulted in a better prediction of the buffet frequency.

Despite buffet having been widely studied in the past 30 years, the mechanism of buffet has not been completely understood yet, in particular regarding the precise nature and behaviour of these upstream and downstream travelling waves. The mechanism by which the DTWs are generated and consequently where they originate in the flow (e.g. in the separated trailing edge area or in the shear layer) remains unclear, although (Lee 1990) (in whose study the flow at the shock foot does not reattach during the whole buffet cycle) and Jacquin et al. (2009) described that the DTWs are created at the shock foot.

A significant contribution to the understanding of the behaviour of the UTWs has been given by the research of Hartmann et al. (2013), who concluded that the UTWs start reaching the shock in the last part of its downstream movement, forcing it to start moving upstream. During the upstream shock movement, the widened area of separated flow reduces the formation of DTWs and consequently of UTWs; therefore, the shock upstream movement is no longer sustained by the UTWs and stops its upstream movement, closing the feedback loop. According to this description, the UTWs are supposed to be created during the whole buffet cycle and with a strength modulated by the buffet frequency. Similarly in D'Aguanno et al. (2019) it was shown that the propagation of UTWs is present during both the upstream and downstream movement of the shock and that they propagate with a velocity that is equal to the speed of sound relative to the flow, which therefore changes throughout the buffet cycle.

An alternative view on buffet was given by Crouch et al. (2007), who described it as a consequence of a global flow instability. The results of this stability analysis accurately predict the buffet onset for $M a$ and $\alpha$ and are in good agreement with experimental data. Although usually the feedback mechanism and the global instability description are considered to be competing theories, for many aspects the feedback description can be seen as the physical mechanism by which the global instability manifests itself.

Since the buffet behaviour over a 2D airfoil is quite periodic, a modal decomposition of the data has been attempted by different research groups, in particular on CFD data. Several studies have been reported in recent years that use Proper Orthogonal Decomposition (POD) to investigate transonic buffet. Szubert et al. (2015) applied POD to scrutinise both the Kelvin Helmholtz (KH) and the Von Kármán (VK) instabilities as possible mechanism by which vortical structures are produced and shed downstream. It has also been demonstrated that the energy associated with the buffet mode is of almost two orders of magnitude greater than the modes associated with $\mathrm{VK}$ and $\mathrm{KH}$ instabilities themselves. In a similar way, (Hall et al. 2000) proposed a reduced-order model (ROM) based on POD for describing transonic flows, showing that by using just a few modes it is possible to obtain a highly accurate reduced-order model in a wide frequency range. An experimental application is presented in Masini et al. (2019), where POD is applied to Pressure Sensitive Paint (PSP) measurements. In Poplingher et al. (2019), a combination of POD and DMD (Dynamic Mode decomposition) is used in order to obtain the modes related to buffet on the RA16SC1 airfoil. Good agreement between the POD and DMD modes was obtained, with the first modes representing the shock oscillation and variation in the separated area with main spectral contribution at the buffet frequency. Higher-order modes at frequency multiples of the buffet show small coherent structures in the shock oscillation area. Ohmichi et al. (2018) used both DMD and POD to study transonic buffet on a swept wing, giving particular attention to the formation of the buffet cells.

It is evident from the literature that the full mechanism of transonic buffet has not been completely resolved yet, especially concerning the formation and behaviour of the downstream travelling waves, but that the use of modal decomposition is a powerful tool to obtain new insight. In this study, POD will also be used to gain further information on the buffet mechanism from the experimental observations. In addition to characterising the main features of the buffet cycle, POD will be used to further construct an efficient reduced-order model (as in Hall et al. (2000)), which allows to extract the small structures in the flow field, like UTWs and vortices being shed in the separated area.

In Sect. 2, a detailed description of the experimental set-up and procedures will be given. This will be followed by a description of the buffet cycle, using schlieren snapshots and PIV phase-averaged velocity fields (Sects. 3, 4). In Sect. 5, the main fluid-dynamics features are analysed by means of POD. Next, in Sect. 6 the propagation of UTWs and of downstream propagating vortices is achieved by making use of POD as a reduced-order model. The 
paper is concluded with a discussion of the buffet mechanism, based on the results obtained (Sect. 7).

\section{Experimental procedures}

\subsection{Facility}

The experiments have been performed in the TST-27, a transonic-supersonic blowdown wind tunnel at TU Delft. The wind tunnel test section is $255 \mathrm{~mm}$ high and $280 \mathrm{~mm}$ wide. Transonic conditions are obtained using a variable choke mechanism downstream of the test section. A technical sketch of the wind tunnel with its main elements is given in Fig. 2 (left)

The experiments have been conducted with a total pressure $p_{0}=2$ bar and a total temperature $T_{0}=288 \mathrm{~K}$; the conditions are summarised in Table 1.

Table 1 Experimental parameters

\begin{tabular}{llll}
\hline Parameter & Symbol & Value & Unit \\
\hline Free stream Mach number & $M a_{\infty}$ & 0.7 & \\
Corrected free stream Mach number & $M a$ & 0.73 & \\
Free stream velocity & $U_{\infty}$ & 225 & $\mathrm{~m} / \mathrm{s}$ \\
Total pressure & $p_{0}$ & 2 & $\mathrm{bar}$ \\
Total temperature & $T_{0}$ & 288 & $\mathrm{~K}$ \\
Chord & $c$ & 0.1 & $\mathrm{~m}$ \\
Span & $b$ & 0.28 & $\mathrm{~m}$ \\
Thickness to chord ratio & $t / c$ & 12.3 & $\%$ \\
Angle of attack & $\alpha$ & 3.5 & $\circ$ \\
Reynolds number based on $c$ & $R e_{c}$ & $2.6 \cdot 10^{6}$ & \\
\hline
\end{tabular}

\subsection{Model}

The airfoil model used is the supercritical OAT15A designed by ONERA and previously used in transonic buffet studies by Jacquin et al. (2009), Deck (2005), Crouch et al. (2009) and several others (see Giannelis et al. 2017). The model of this investigation has a span of $280 \mathrm{~mm}$, chord $(c)$ of $100 \mathrm{~mm}$ (Fig. 2, right) and a thickness-to-chord ratio $(t / c)$ of $12.3 \%$. For production reasons, the trailing edge has a thickness of $0.75 \% c$. In order to ensure a turbulent boundary layer, a transition trip has been applied on the suction side at $7 \%$ of the chord of the airfoil, which was obtained by using homogeneously distributed Carborandum $500(\mathrm{SiC})$ particles (with a size of $0.014 \mathrm{~mm}$ ).

The airfoil model spans the full width of the test section and was mounted on the side windows of the wind tunnel, which provide optical access from both sides, see Fig. 2 (right).

The experiments have been performed at a free stream $M a=0.7, \alpha=3.5^{\circ}$ and $R e_{c}=2.6 \cdot 10^{6}$ (based on the chord of the airfoil). In previous experiments performed in the same wind tunnel on the same airfoil (Schrijer et al. 2018), buffet has been demonstrated to be fully developed for these conditions. When correcting the value of the Mach number for the blockage caused by the presence of the airfoil in the wind tunnel, a value of $M a=0.73$ is obtained. The values of $M a=0.73$ and $\alpha=3.5^{\circ}$ for the most developed buffet condition agree with those mentioned in the literature for the same airfoil (Jacquin et al. 2009).

\subsection{Experimental measurements techniques}

High-speed schlieren and particle image velocimetry (PIV) have been applied as flow diagnostic techniques to investigate the flow dynamics.

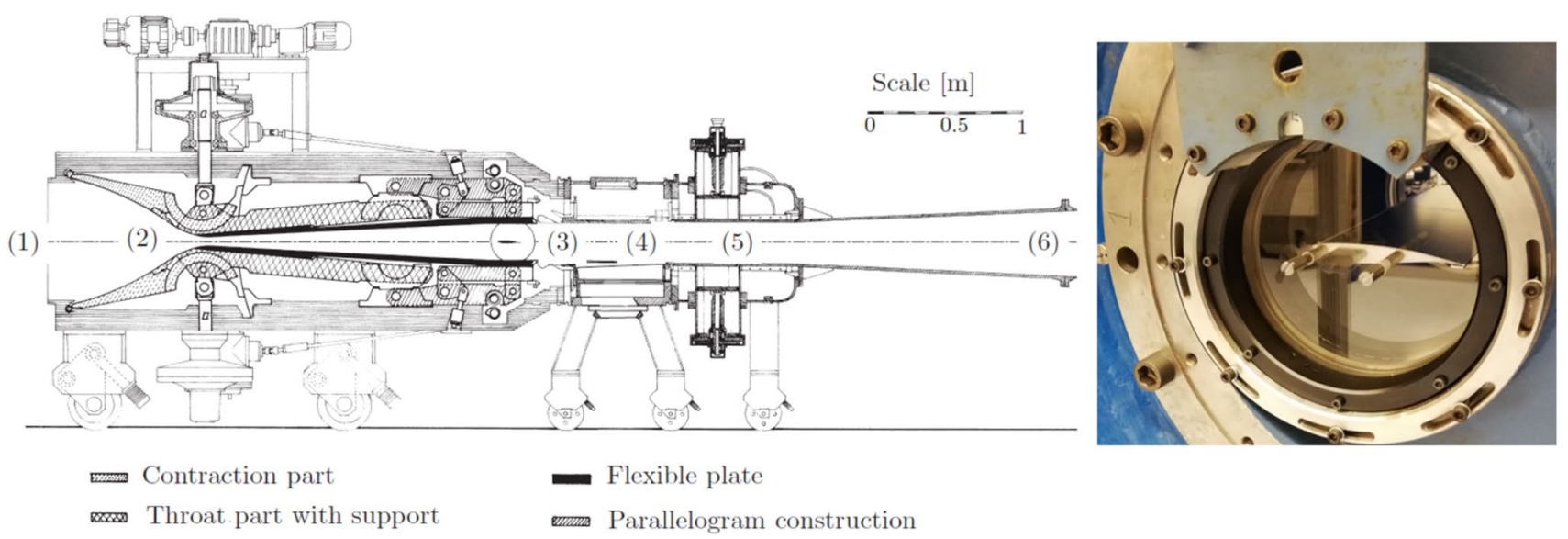

Fig. 2 Technical drawing of TST 27 [settling chamber (1), nozzle (2), test section and model (3), changeable cart (4), choke (5) and diffuser (6)] (left) and OAT15A installed in the test section (right) 
The schlieren set-up employs a conventional z-configuration using a pinhole diameter of $2 \mathrm{~mm}$ and a LED-based continuous illumination system. An Imager Pro high-speed camera has been used for image acquisition, with the sensor cropped to $912 \times 816$ pixels $(7.6 \mathrm{pix} / \mathrm{mm})$ to achieve an acquisition frequency of $5 \mathrm{kHz}$ and a total acquisition time of $2 \mathrm{~s}$. Since the buffet frequency is $160 \mathrm{~Hz}$, the selected frequency is sufficient to resolve the shock position in time, obtaining an average of more than 30 images per buffet cycle. The exposure time of the camera was set to $15 \mu \mathrm{s}$ which essentially eliminates shock motion blurring.

In order to characterise the buffet phenomenon in more detail, a high-speed 2-component PIV investigation was performed using the set-up shown in Fig. 3 (right), comprising a high-speed laser (Continuum MESA PIV 532-120-M) and two Photron Fastcam SA-1 cameras. The image acquisition frequency was $4650 \mathrm{~Hz}$ in double pulse mode $(\delta t=3 \mu \mathrm{s})$ with a resolution of $1024 \times 640$ pixels and an acquisition time of $0.94 \mathrm{~s}$. The two cameras were placed on opposite sides of the wind tunnel in order to have overlapping fields of views (FOVs) as shown in Fig. 3 (left). The measurement plane is located in the mid-span of the airfoil and oriented in the streamwise direction, with both FOVs having a size of $50 \times 30 \%$ of the chord $(50 \times 30 \mathrm{~mm}) . F O V_{1}$ starts at $0.26 \% \mathrm{c}$ from the leading edge of the airfoil, while $\mathrm{FOV}_{2}$ stars at $72 \% \mathrm{c}$.

The laser used (Nd:YAG) is a dual-cavity laser, operated in double pulse mode with a pulse duration of 150 $\mathrm{ns}$ and a wavelength of $532 \mathrm{~nm}$. The laser system illuminates the seeding particles, which are liquid droplets of DEHS (Di-Ethyl-Hexyl-Sebacat), which have been demonstrated by Ragni et al. (2011) to follow the flow accurately, having a Stokes number greater than one only in the shockwave location, considering a particle relaxation time of $2 \mu \mathrm{s}$. The laser sheet, which had a thickness of approximately $1.5 \mathrm{~mm}$, was generated by a light sheet optics probe inserted from the side of the wind tunnel and located downstream of the test section as indicated in Fig. 3 (right).

The cameras and lasers were synchronised using a LaVision high-speed controller (art. 1108075). PIV image acquisition and processing were done using LaVision Davis 8.4.0.

\subsection{Data processing}

To reduce the effect of laser light reflections which are present on the surface of the airfoil and at the trailing edge, pre-processing of the particle images is carried out by means of an 11 image time-minimum subtraction. Thereafter, by means of a cross-correlation procedure between the first and the second pulse for each camera, the velocity field is obtained. The cross-correlation is computed with a multi-pass approach: two passes with a window size of $64 \times 64$ pixels and two passes with a final circular window size of $24 \times 24$ pixels. In both cases, an overlap of $75 \%$ has been selected obtaining a final vector spacing of $0.3 \%$ of the chord $(0.3 \mathrm{~mm})$.

For both schlieren and PIV, additional processing has been carried out mostly in Matlab. In particular for PIV, a validation of the velocity vectors has been performed in order to correct outliers. A first detection of outliers was based on discarding vectors when their values were not included in \pm 3 standard deviations with respect to the average value of both the velocity components. Those outliers were substituted with an interpolation of the neighbouring vectors. It should be noted that this operation is not effective for large patches of outliers, which occur due to intermittent seeding particles. To resolve this problem, an interpolation in time instead of in space has been applied as well.
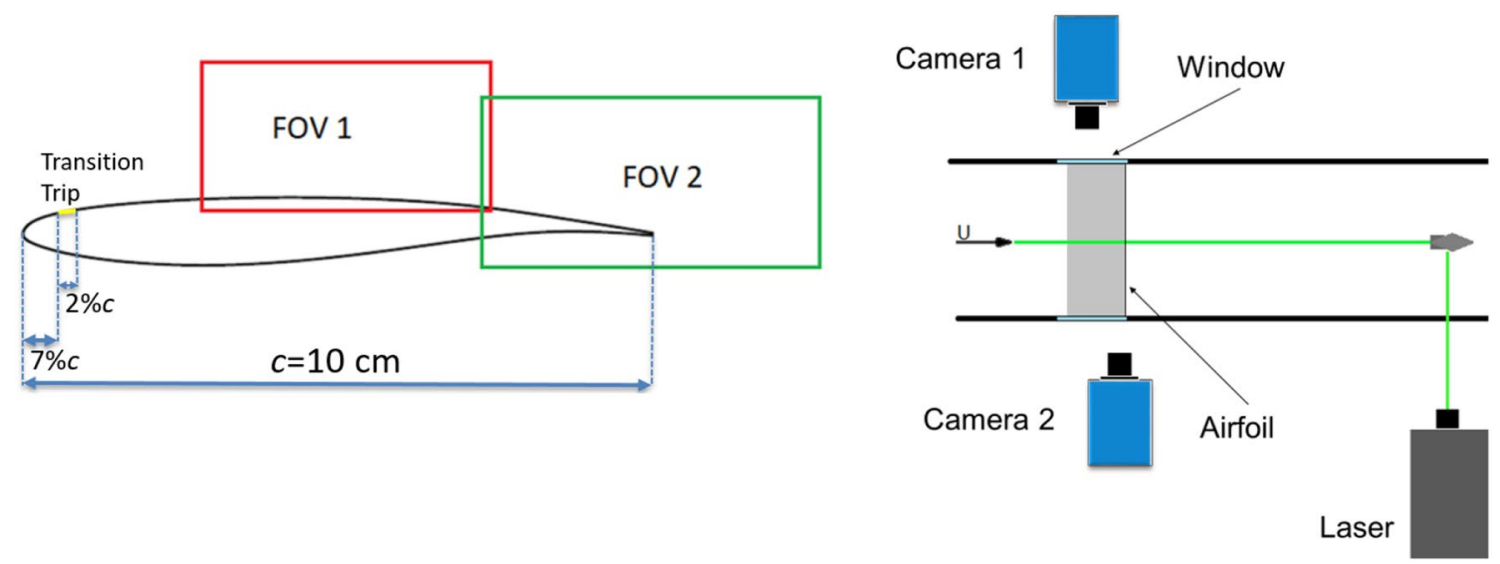

Fig. 3 FOVs on the OAT15A airfoil (left) and sketch of PIV set-up (right) 


\subsection{Uncertainty analysis}

All the experimental data are affected by uncertainties and their quantification is of upmost importance, especially when assessing flow property fluctuations.

A first source of uncertainty on the mean value is the statistical convergence uncertainty due to the finite ensemble size that for the two components of velocity can be obtained as (Benedict and Gould 1996):

$\varepsilon_{\bar{u}}=\frac{\sqrt{u^{\prime 2}}}{\sqrt{N}}$

$\varepsilon_{\bar{v}}=\frac{\sqrt{v^{\prime 2}}}{\sqrt{N}}$

where the numerator contains the corresponding standard deviation, whereas $N$ is the number of uncorrelated images taken to compute the average (ensemble size). However, under the present conditions the velocity vectors cannot be considered uncorrelated because of the high sampling frequency $(4650 \mathrm{~Hz})$ with respect to the characteristic frequency that governs the fluid dynamics $(160 \mathrm{~Hz})$. A first conservative estimation of the uncertainty could be obtained substituting instead for $N$ the number of buffet cycles.

In order to obtain a more accurate estimate, an effective number of uncorrelated images should be considered by using a correction factor. Starting from the uncertainty definition of a variable (here $u$ is taken as example), it is possible to obtain that (Coleman and Steele 2009; Sciacchitano and Wieneke 2016):

$\varepsilon_{\bar{u}}^{2}=\sum_{i=1}^{N} \sum_{j=1}^{N} \frac{1}{N^{2}} \rho\left(u_{i}, u_{j}\right) \sigma_{x}^{2}$

where $\rho$ is computed as the autocorrelation in time of each velocity component, and it is evaluated in each spatial point. Rearranging, $\rho$ can be expressed as a function of the time separation between samples $n \Delta t$ (where $n$ indicates different time steps and $\Delta t=1 / 4650 \mathrm{~s}$ the time separation between consecutive snapshots):

$\varepsilon_{\bar{u}}^{2}=\frac{\sum_{n=-\infty}^{\infty} \rho(n \Delta t)}{N} \sigma_{u}^{2}=\frac{\sigma_{u}^{2}}{N_{\text {eff }}}$

As suggested in Sciacchitano and Wieneke (2016), the summation $\sum_{n=-\infty}^{\infty} \rho(n \Delta t)$ is truncated when $\rho(n \Delta t)$ reaches zero for the first time. The resulting value of $N_{\text {eff }}$ depends on the location in the FOV, having higher values in the shock location and in the oscillating separated area. In Table 2, the resulting maximum uncertainty values obtained in the FOV are given for both the velocity components. It is worthwhile
Table 2 Uncertainty values

\begin{tabular}{lll}
\hline Uncertainty source & Error & Unit \\
\hline Statistical $\left(\varepsilon_{u}\right)$ & $\leq 6.7$ & $\mathrm{~m} / \mathrm{s}$ \\
Statistical $\left(\varepsilon_{v}\right)$ & $\leq 2.2$ & $\mathrm{~m} / \mathrm{s}$ \\
Cross-correlation $\left(\varepsilon_{c c}\right)$ & $\leq 6.3$ & $\mathrm{~m} / \mathrm{s}$ \\
Spatial resolution $\left(\varepsilon_{s r}\right)$ & $\leq 1$ & $\%$ \\
Particle slip $\left(\varepsilon_{\text {slip }}\right)$ & $\leq 60$ & $\mathrm{~m} / \mathrm{s}$ \\
Line of sight effect $\left(\varepsilon_{\text {schlieren }}\right)$ & $\leq 3$ & $\mathrm{~mm}$ \\
\hline
\end{tabular}

to mention that the values of $\frac{N}{N_{e f f}}$ in the region of interest are in the order of seven images, indicating that subsequent snapshots remain correlated for about $1 / 4$ of the buffet cycle.

Apart from the statistical uncertainties, there are additional sources of uncertainty on the individual velocity values which will be treated in this section.

An important contribution is due to the fact that PIV is affected by the finite time response of the seeding particles, in particular in correspondence of the shockwave. This results in a particle slip velocity described by Melling (1997):

$\varepsilon_{\text {slip }}=\vec{U}_{\text {slip }} \approx \tau_{p} \cdot \vec{a}_{p}$

The uncertainty value is obtained by approximating the acceleration term and considering that the DEHS particles which have been used have a response time of approximately $\tau_{p}=2 \mu$ s (Ragni et al. 2011).

Also, the cross-correlation procedure used in PIV brings an uncertainty estimated as (Humble 2009):

$\varepsilon_{c c}=\frac{\varepsilon_{c o r r}}{M \delta t}$

where $\mathrm{M}$ is the magnification factor (with $\mathrm{M}=0.4$ in this study) and $\delta t$ the laser pulse separation. For planar PIV, it is assumed $\varepsilon_{\text {corr }}=0.1$ pixel.

Finally, there is an uncertainty due to the fact that the use of a specific window size (WS) allows the resolution of the flow only up to a certain scale (with corresponding wavelength $\lambda$ ), as has been described by Schrijer and Scarano (2008) as:

$\varepsilon_{s r}=\frac{u}{u_{0}}=\operatorname{sinc}\left(\frac{W S}{\lambda}\right)$

This effect is reduced in the case of a multi-step correlation, and considering that typically the smallest resolvable scale in the flow has twice the size of the WS (see Sun 2014; de Kat and van Oudheusden 2012), a value of $\varepsilon_{s r}<1 \%$ can be estimated.

Furthermore, also the schlieren data are affected by uncertainty. In this study, these images are analysed mainly 
to obtain the instantaneous shock position. Therefore, it must be taken into account that the density gradients visualised in a schlieren image are integrated along the entire span of the airfoil. For this reason, when a shock is not perpendicular to the light propagation vector, the shock is displayed in the schlieren image from its projected area, and therefore, it occurs thicker than it really is. The error can be evaluated as half of the thickness of the projected shock $\left(t_{S W}\right)$, considering the real local thickness of the shockwave negligible.

$\varepsilon_{\text {schlieren }}=\frac{t_{S W}}{2} \approx 3 \mathrm{~mm}$

In the following table, different sources of uncertainty are collected. The highest value is associated with the particle slip. This value is computed in the shock region, which contains the largest velocity gradients. In particular, this estimation of the slip velocity in the shock region is highly influenced by the value of $\tau_{p}$ for DEHS particles in combination with the large particle deceleration across a shock.

\section{Flow dynamics}

In this section, the unsteady behaviour of transonic buffet will be studied starting from the instantaneous schlieren images by examining a typical buffet cycle. The cycle is divided into different phases in accordance with the shock position. The most relevant phases are those with the shock in the most upstream and downstream position as well as the two in between, i.e. with the shock in an intermediate position, travelling either upstream or downstream. The schlieren images corresponding to these phases are shown in Fig. 4 for the time instants that are indicated in Fig. 5 (left) with an ' $*$ '. In the images, the phases are numbered 1, 3, 5 and 7 , respectively, in order to correspond to the phases that are defined in Sect. 4.

The supersonic region is clearly visible on the suction side including the terminating shock. Close to the leading edge, a prominent stationary wave appears, which is associated with the presence of the transition trip at the $7 \%$ of the chord. For reference, in all the snapshots the absolute most upstream $(27 \% \mathrm{c})$ and the most downstream $(55 \% \mathrm{c})$ positions of the SW are indicated, with a red and a green vertical line, respectively. In the image on the top left, the shockwave is in the most upstream position, around $27 \%$ of the chord. When the shock is at this location, there is a large separated region which starts at the shock foot and develops till the trailing edge.

Continuing (figure in the top right), the shockwave starts its downstream movement, and due to the decreasing relative velocity with respect to the flow it becomes weaker. This is confirmed by the fact that the separated area is smaller and develops only downstream of the SW oscillation range. Both in this and the previous phase, the presence of UTWs is observed, close to the vertical green line, as quasi-normal black lines.

In the following phase (bottom left), the shock reaches the most downstream position of this particular buffet cycle, which is at approximately 53\%c. Although the separated region is still relatively small in the direction normal to the
Fig. 4 Instantaneous schlieren images in four main phases; the red and the green bars indicate, respectively, the most upstream and downstream position of the SW among all the images. Top left: $\mathrm{SW}$ is in the most upstream position. Top right: SW travelling downstream. Bottom left: SW in the most downstream position. Bottom right: SW travelling upstream
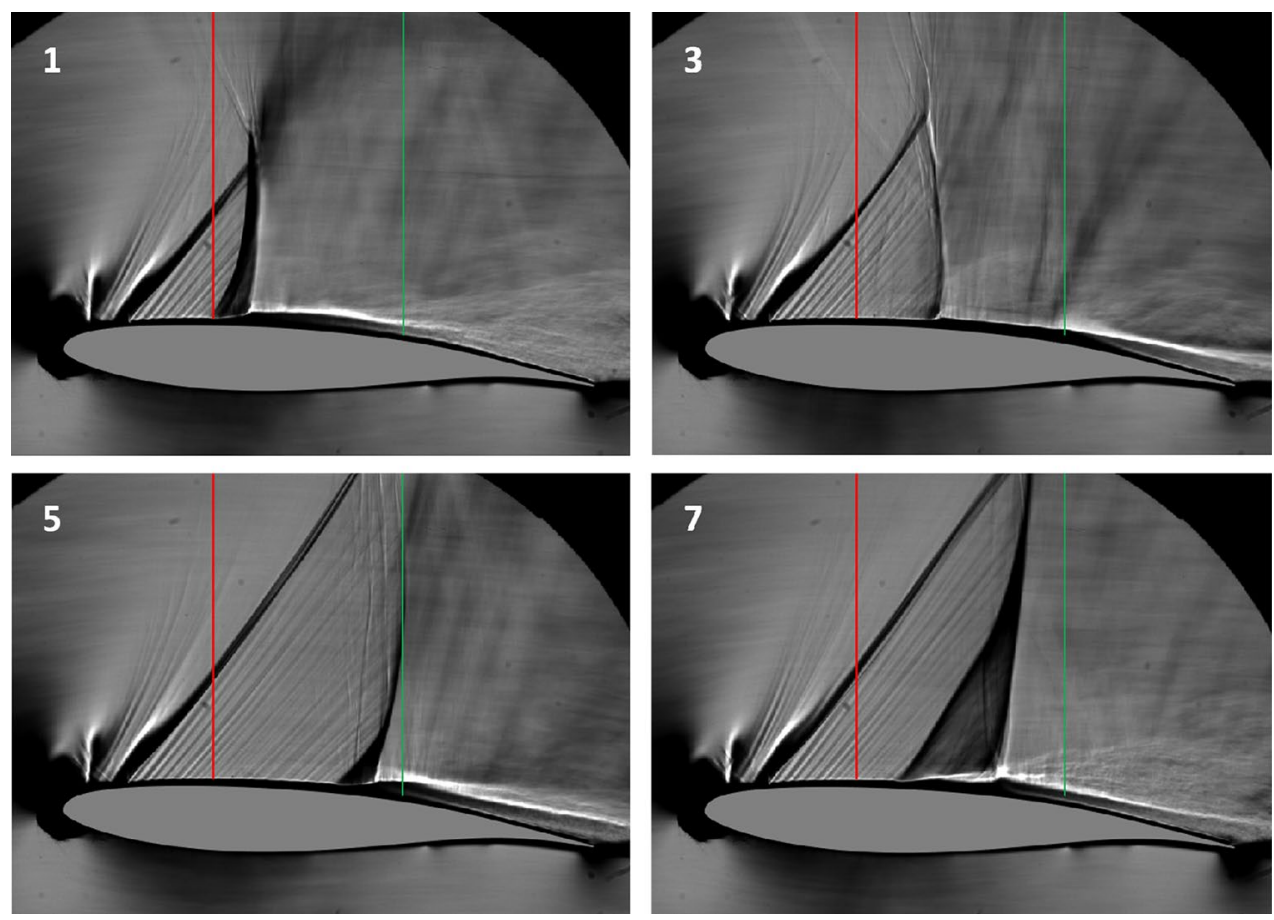

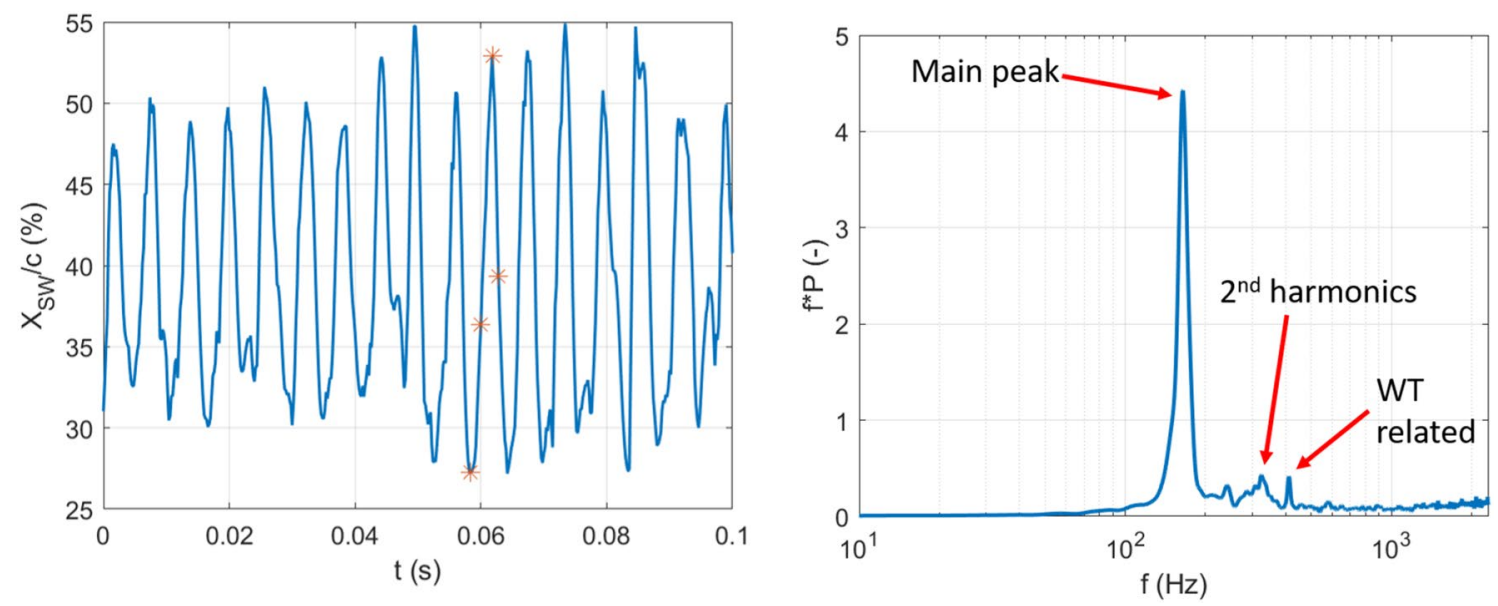

Fig. 5 Temporal variation in shock position (left) (the * corresponds to the snapshots shown in Fig. 4). Pre-multiplied PSD related to SW position (right)

airfoil upper surface, it is again triggered at the shock foot in this phase.

Next, (bottom right) the shockwave moves upstream becomes stronger and reaches the situation in which the separated area is the largest. The shock appears wider than in the previous schlieren image. This is due to the simultaneous presence of a $\lambda$-shock structure (close to the surface of the airfoil) and 3D effects.

\subsection{Spectral analysis}

The shock location as a function of time is extracted from the schlieren images by evaluating the maximum horizontal gradient of the image intensity. In order to reduce the random errors related to the shock detection, the shock position obtained along five parallel horizontal lines was averaged. The lines considered have been taken at a distance of $0.1 \mathrm{c}$ $(1 \mathrm{~cm})$ from the airfoil chord in order to avoid possible double detections close to the surface of the airfoil because of the $\lambda$-shock structure. A similar shock detection procedure was applied to the instantaneous PIV images based on the streamwise gradient of the $u$ component of the velocity.

In Fig. 5 (left), the shock position obtained from the schlieren images is plotted in time showing an oscillation from a most upstream to a most downstream position with nearly constant period; however, the phenomenon cannot be considered perfectly periodic as secondary low-frequency phenomena are present as well. When considering all the snapshots, an average shock position $\left(X_{S W}\right)_{a v g}=37.7 \% \mathrm{c}$ and a relative standard deviation $\left(X_{S W}\right)_{s t d}=6.7 \% c$ are obtained.

To characterise the shock oscillation, a spectral analysis is performed by evaluating the power spectral density (PSD) of the shock position, which is obtained as the Fourier Transform of the autocorrelation $\mathrm{r}(\mathrm{k})$ of the signal $x_{S W}(t)$ :
$\operatorname{PSD}(f)=\sum_{n=-\infty}^{\infty} r(k) \cdot e^{-i 2 \pi k n}$

where $\mathrm{r}(\mathrm{k})$ is defined as:

$r(k)=E\left\{X_{S W}(t) X_{S W}^{*}(t-k)\right\}$

In order to reduce the noise in the PSD, the Welch method (Welch 1967) has been used, dividing the ensemble size in bins with $50 \%$ overlap of 625 samples each and averaging the corresponding PSDs. The maximum resolved frequency is $2500 \mathrm{~Hz}$ and $2325 \mathrm{~Hz}$ for the schlieren and PIV measurements, respectively, with a resolution of $1 \mathrm{~Hz}$.

In Fig. 5 (right), the PSD $(P(f))$ is shown, in pre-multiplied form, $f \cdot P(f)$ on a frequency $\log$ scale. From the plot, the presence of a dominant peak at $160 \mathrm{~Hz}$ is evident. This value corresponds to a Strouhal number $S t=\frac{f \cdot c}{U_{\infty}}=0.07$ which is in perfect agreement with the results obtained by Jacquin et al. (2009) for experiments carried out for similar conditions and airfoil geometry. In addition to a 2nd-harmonics contribution, there is also an additional secondary peak at $410 \mathrm{~Hz}$ which is associated with the characteristic noise of the wind tunnel. The apparent increase in $f \cdot P(f)$ for $f>1 \mathrm{kHz}$ is associated with the uncertainty in the shock detection.

\section{Phase-averaged flow description}

\subsection{Time-average velocity field}

A more quantitative description of the buffet flow field has been obtained from the PIV velocity data, which will be used from this section on. 

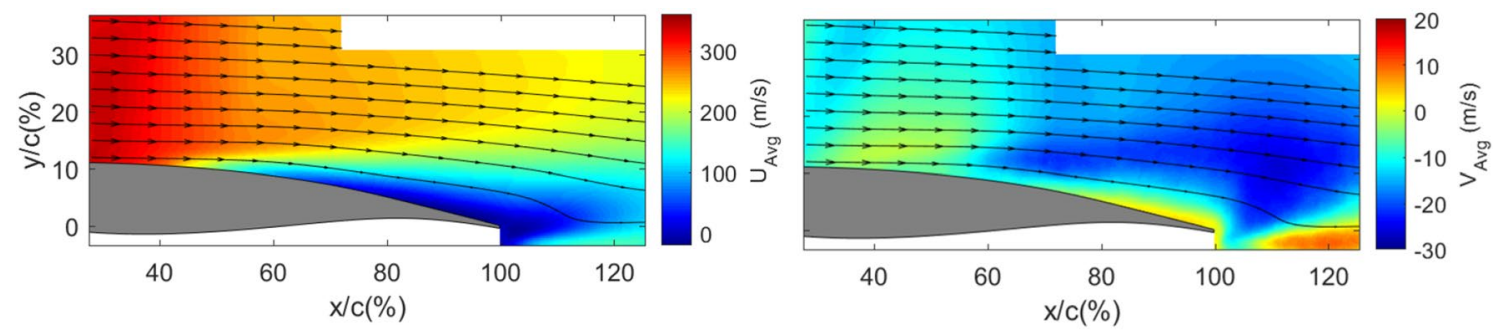

Fig. 6 Average velocity field for horizontal (left) and vertical component (right)

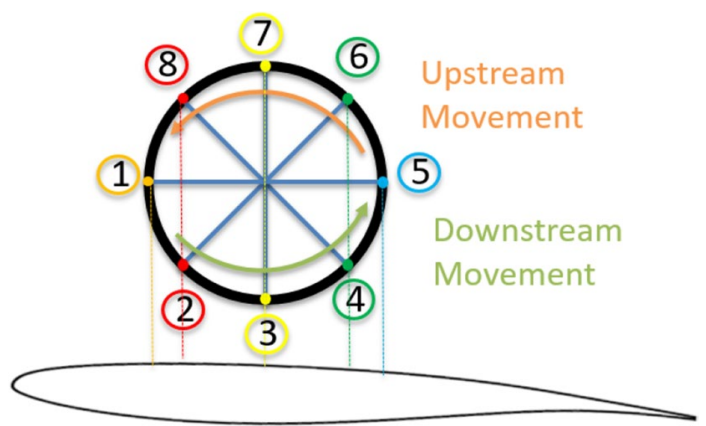

Fig. 7 Definition of buffet cycle phases, in accordance with shock position and movement

Figure 6 shows the time-average velocity distribution for both the horizontal and the vertical velocity components. For both velocity fields, streamlines are included in order to illustrate the flow topology. The visualisation of the horizontal velocity components reveals the supersonic region upstream of the shock, the shear layer and the separated area over the rear part of the airfoil. From the vertical velocity component, a region of increased velocity in the shock foot oscillation area (between 30 and 55\%c) appears as well as a region of positive velocity (upwash) in the separated trailing edge area.

\subsection{Phase definition}

Evidently, the time-average representation of the flow does not allow the visualisation of various elements of the dynamics of shock buffet. Therefore, a phase average visualisation approach is applied instead. For obtaining the phase average, the triple decomposition is defined as:

$\vec{u}=\vec{u}_{\text {avg }}+\vec{u}_{p e r}+\vec{u}_{t u r b}$

where $\vec{u}_{\text {avg }}$ is the average contribution, $\vec{u}_{p e r}$ represents the periodic component and $\vec{u}_{t u r b}$ is the quasi-random fluctuating contribution. In the following analysis, the phase averaged velocity field is considered, which is taken as the sum of the mean and the periodic contributions $\left(\vec{u}_{p e r}+\vec{u}_{a v g}=\vec{u}_{p h s}\right)$. For this analysis, the buffet cycle is divided into eight phases
Table 3 Statistical uncertainty per phase

\begin{tabular}{llll}
\hline Statistical errors & Error Vx $(\mathrm{m} / \mathrm{s})$ & Error Vy $(\mathrm{m} / \mathrm{s})$ & $\begin{array}{l}\text { Num- } \\
\text { ber of } \\
\text { images }\end{array}$ \\
\hline 1st Phase $\left(\varepsilon_{P h s 1}\right)$ & $\leq 4.9$ & $\leq 1.6$ & 1110 \\
2nd Phase $\left(\varepsilon_{P h s 2}\right)$ & $\leq 7.6$ & $\leq 2.5$ & 476 \\
3rd Phase $\left(\varepsilon_{P h s 3}\right)$ & $\leq 8.4$ & $\leq 2.7$ & 394 \\
4th Phase $\left(\varepsilon_{P h s 4}\right)$ & $\leq 8.0$ & $\leq 2.6$ & 427 \\
5th Phase $\left(\varepsilon_{P h s 5}\right)$ & $\leq 5.7$ & $\leq 1.9$ & 839 \\
6th Phase $\left(\varepsilon_{P h s 6}\right)$ & $\leq 9.7$ & $\leq 3.1$ & 295 \\
7th Phase $\left(\varepsilon_{P h s 7}\right)$ & $\leq 10.3$ & $\leq 3.3$ & 260 \\
8th Phase $\left(\varepsilon_{P h s 8}\right)$ & $\leq 8.5$ & $\leq 2.7$ & 387 \\
\hline
\end{tabular}

according to the position and direction of movement of the shock, such that the first phase corresponds to the most upstream position. The fifth phase then corresponds to the most downstream shock location, as shown by the sketch in Fig. 7.

In Table 3, the number of images belonging to each phase is reported together with the corresponding statistical uncertainties, which are here obtained using Eqs. 1 and 2 (it is assumed that the snapshots belonging to each phase are uncorrelated). Once the phases have been defined and the images associated with the corresponding phase bins (group of images belonging to the same phase) have been identified, it is possible to quantitatively characterise different phases by looking at the probability density function (pdf) associated with the shock position $p\left(X_{S W} / c\right)$ and shock velocity $p\left(V_{S W}\right)$ as shown in Fig. 8. The pdf has been obtained using bins with a bandwidth of $2 \%$ of the chord for the shock position and of $2 \mathrm{~m} / \mathrm{s}$ for the shock velocity. The values of the pdf for different phases are normalised by the total number of snapshots.

Starting with the shock position (Fig. 8, left), it is observed that the pdf associated with all the shock positions (indicated as "all phases") is significant in the region that ranges from 25 to $55 \%$ of the chord, having two local maxima upstream and downstream of the average shock position, which is at $40 \%$ of the chord. These results were expected since the shock position is oscillatory therefore having a pdf 

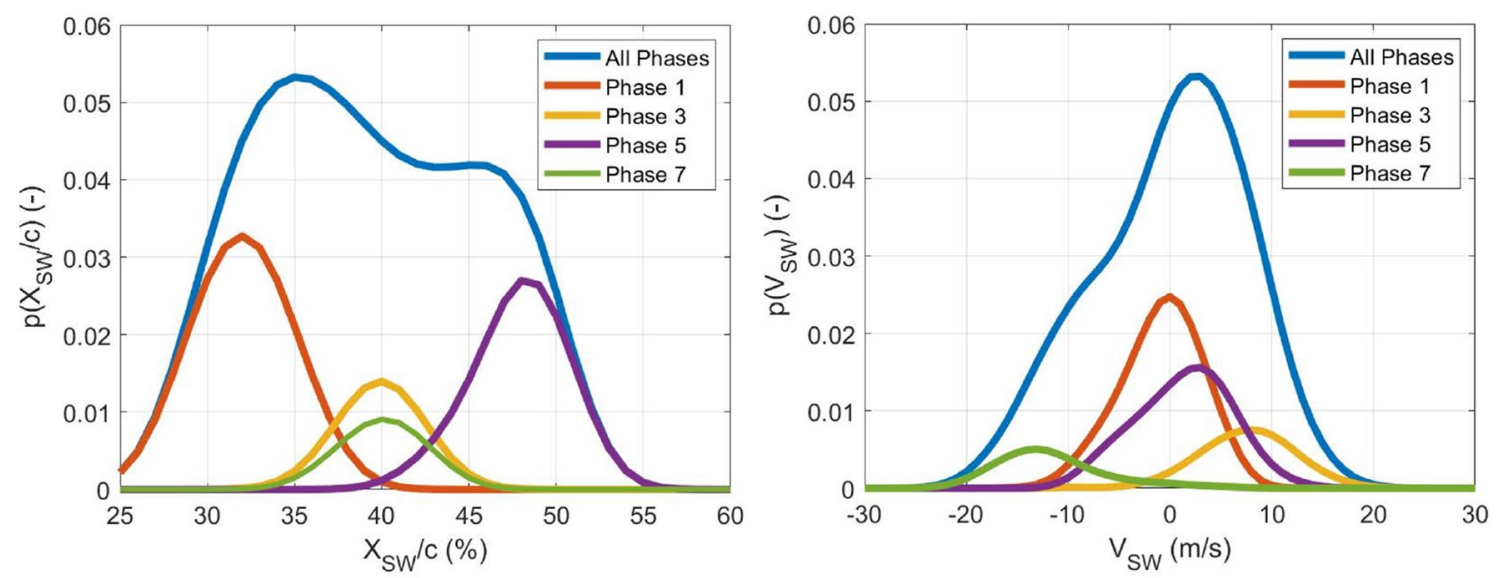

Fig. 8 Pdf of shock position (left) and velocity (right)

similar to that of a sinusoidal signal. This result is clarified when looking at the pdf of the shock velocity (Fig. 8, right) for which in phases 1 and 5 (most extreme shock positions) the maximum likelihood is close to zero, while for phase 3 and phase 7 where the maximum likelihood is, respectively, positive and negative. In particular, in absolute terms the higher velocity is reached during phase 7 with an average velocity of $-12 \mathrm{~m} / \mathrm{s}$. Going back to the pdf of the shock position, there is quite a symmetrical behaviour between different phases in terms of shock location. In phase 3 and phase 7 , as expected from the way they have been defined, the shock is located in exactly the same region, while the region of interest of phases 1 and 5 (most upstream and downstream position) is equally far from the average shock position. It should be noted that the pdf relative to the shock position in phases 3 and 7 differs in magnitude only because of the fewer number of images present in phase 7 (in which the shock moves faster, and therefore, less images are captured).

The pdf associated with all images ("all phases") has an asymmetrical shape which indicates that the shock tends to dwell a longer time in the forward position. Furthermore, the number of images belonging to the each phase (see Table 3 ) suggests that the downstream motion is on average slower than the upstream motion. This is confirmed by $p\left(V_{S W}\right)$, which is skewed towards positive values.

\subsection{Phase average velocity fields}

Figure 9 shows the phase average of the $u$ (left) and of the $v$ (right) component of the velocity for the 1st, 3rd, 5th and 7th phase (i.e. the same phases as shown in Fig. 4). Streamlines are included to show the local flow direction.

In the first phase, the shock is in the most upstream position and has a slightly oblique orientation. Correspondingly, an increase in vertical velocity at the shock foot is observed, resulting from the flow deflection associated with the upward displacement of the shear layer, similar to the corresponding schlieren visualisation in Fig. 4 (Top left). More downstream it is possible to observe the trailing edge separated area, which extends upstream till $60 \%$ of the chord. The separation can also be observed in the vertical velocity with an increase in the v-component of the velocity at the trailing edge.

In the third phase (second row in Fig. 9), the shock is visualised during its downstream movement, and since the velocity of the shockwave relative to the flow is the lowest in this phase, the separated region is smaller compared to the previous case, as it is clear from both the $u$ and the $v$ velocity component. No increase in vertical velocity is observed at the shock foot, and thus, the shockwave is more normal with respect to the flow than in the previous phase.

In the fifth phase (third row in Fig. 9), the shockwave has reached the most downstream location. Because of this, the supersonic region is larger as the velocity field is accelerated for a longer distance upstream of the shockwave. In this phase, the shock starts moving again upstream and therefore increases in strength. This is reflected in the fact that the size of the separated region has increased again.

In the seventh phase (fourth row in Fig. 9), as was already mentioned before, the shock has the largest velocity with respect to the flow, and therefore, it is expected to be the strongest in this stage. The shock appears more oblique and the separated area at the shock-foot is wider, as both the $u$ and the $v$-component of the velocity indicate.

After this phase, the shockwave will complete the buffet cycle reaching again the most upstream position.

\section{Modal description of flow features}

While in Sect. 3.1 a spectral analysis of the shock motion based on the schlieren images was presented, Fig. 10 shows a spectrogram of the u-component of the velocity for a 

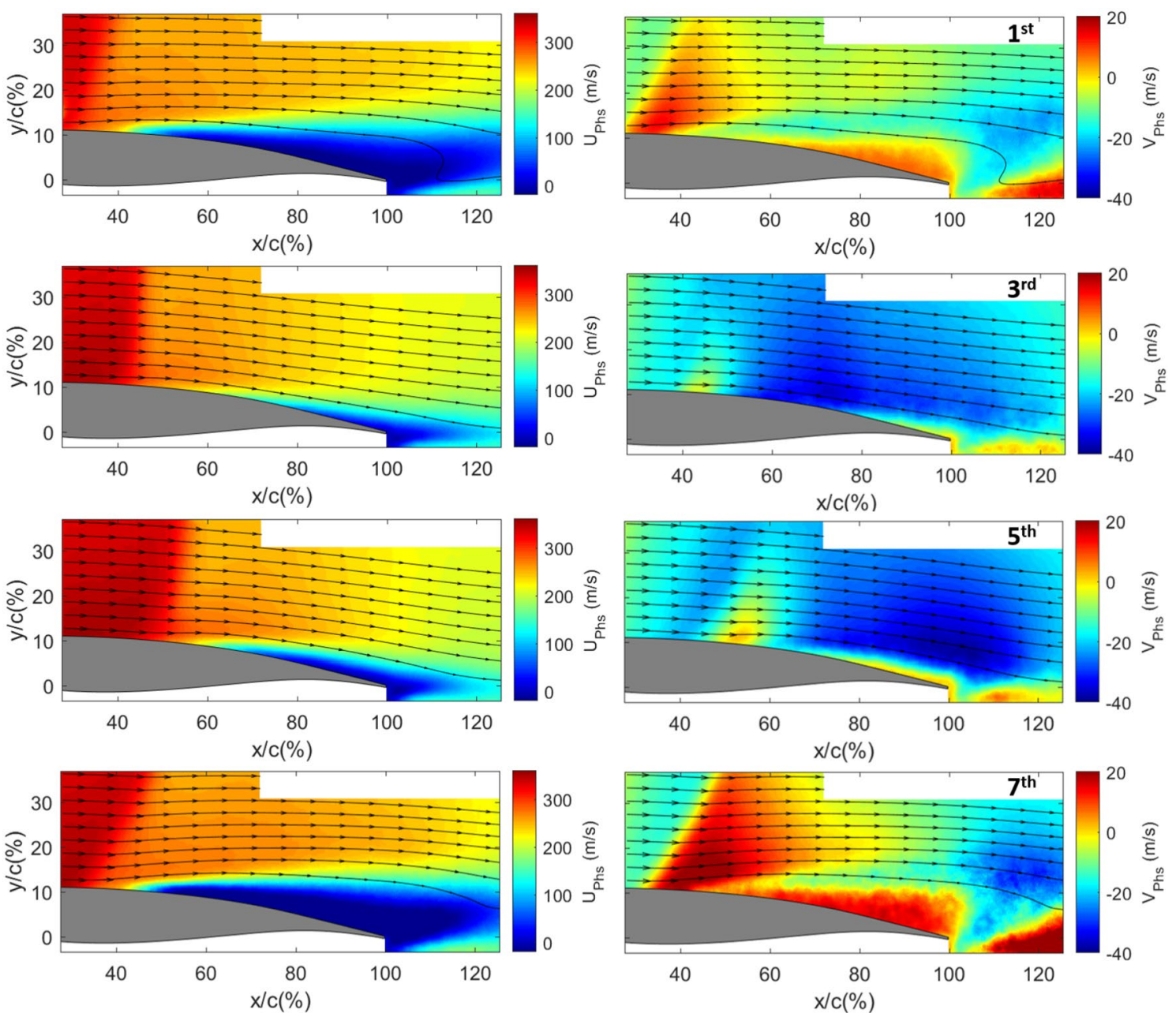

Fig. 9 PIV phase average for $u(l e f t)$ and $v$ (right) velocity component for the 1st, 3rd, 5th and 7th phase (going from top to bottom)

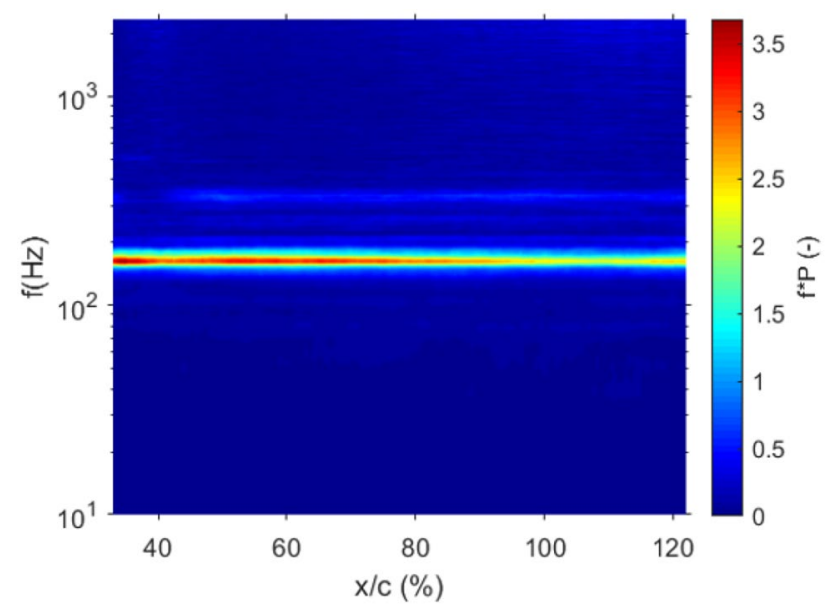

Fig. 10 Spectrogram of the horizontal component of velocity on a horizontal line at $\mathrm{y} / \mathrm{c}=12 \% \mathrm{c}$ horizontal line of the PIV FOV at $y / c=12 \%$ (see $y$-axis' scale in Fig. 6), with the spectrogram expressed in terms of the pre-multiplied PSD.

It is evident that there is a strong contribution at $160 \mathrm{~Hz}$ in the shock oscillation region and in the separated area, while a contribution at $320 \mathrm{~Hz}$ appears mainly downstream of the average shock position (around $45 \% \mathrm{c}$ ). Contributions at higher frequencies are not relevant in the whole spectrogram. The dominant frequencies extracted from the spectrogram agree with the ones that were obtained for the shock position from the schlieren snapshots (Fig. 5 (right)). However, from this analysis it is not evident which kind of flow structures is associated with the peaks at $160 \mathrm{~Hz}$ and $320 \mathrm{~Hz}$. Therefore, a more detailed investigation based on a modal decomposition of the PIV flow field data was undertaken.

\subsection{POD analysis}

The phase averaging procedure that is discussed in Sect. 4 can be considered as a form of reduced-order modelling, 
based on shock position and motion. For a more rigorous approach, a representation of the time-varying flow dynamics associated with buffet is made based on Proper Orthogonal Decomposition (POD), by which the most energetic modes are extracted from a signal with particular spatial and temporal dynamics. In this investigation, the so-called snapshot-POD (see Sirovich 1987 for more information) has been used since it is particularly efficient when the number of spatial coordinates is greater than the number of snapshots as in the case of this study.

Considering $\vec{u}(\vec{x}, \mathrm{t})$ as a function of both time $(t)$ and spatial coordinates $(\vec{x}(\mathrm{x}, \mathrm{y}, \mathrm{z}))$, POD allows to describe the fluctuation component $u^{\prime}$ with a spatio-temporal decomposition

$\vec{u}(\vec{x}, t)=\vec{u}_{\text {avg }}(\vec{x})+\vec{u}^{\prime}(\vec{x}, t)=\vec{u}_{\text {avg }}(\vec{x})+\sum_{j=1}^{N} \sqrt{\lambda_{j}} \cdot c_{j}(t) \cdot \vec{\phi}_{j}(\vec{x})$

where $c_{j}(t)$ and $\vec{\phi}_{j}(\vec{x})$ are, respectively, the orthonormal time coefficients and the orthogonal spatial POD functions, while $N$ is the total number of modes which is equal to the total number of snapshots $(\mathrm{N}=4325) . \lambda_{j}$ are the POD eigenvalues of the problem which give the kinetic energy associated with each POD mode.

Each spatial mode can be obtained as:

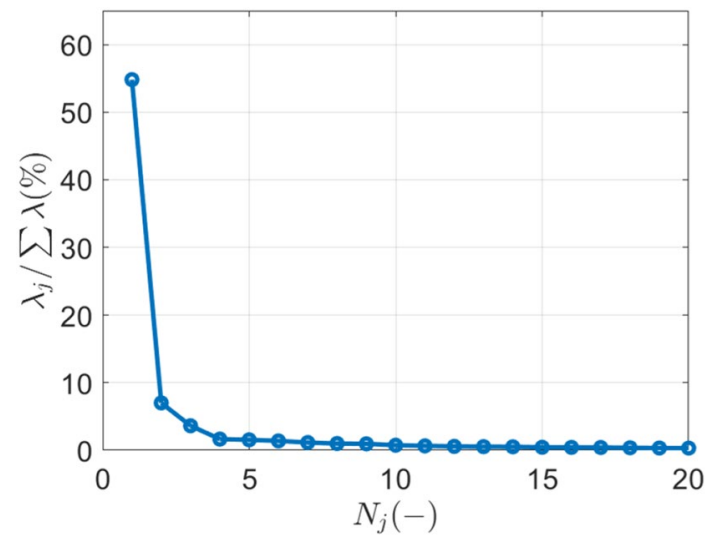

Fig. 11 Energy fraction associated with the first 20 POD modes

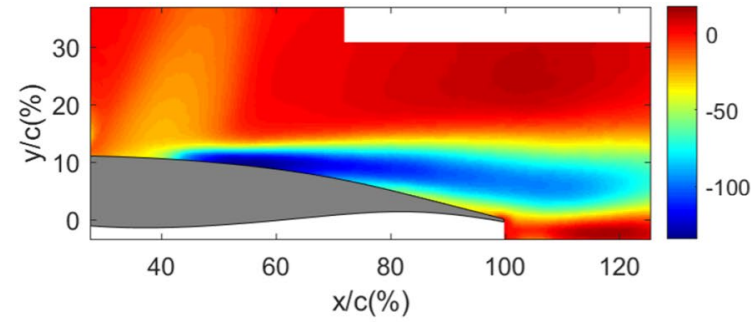

$U_{\text {mode }}(j)=\sqrt{\lambda(j)} \cdot \vec{\phi}_{j}(\vec{x})$

More details about POD and snapshot-POD can be found in Arányi et al. (2013).

Equation 12 may be used to reconstruct the velocity field using a subset of the modes $(M<N)$, therefore realising a reduced-order reconstruction. This reconstruction is used in Sects. 5.4.3 and 6.

\subsection{POD energy spectrum}

By using the POD algorithm, the eigenvalues associated with different modes are obtained and shown in Fig. 11 in terms of energy fraction for the first 20 modes. The energy fraction is computed as the ratio of the eigenvalue of each mode $\lambda_{j}$ and the cumulative energy of all the modes $\sum_{j=1}^{N} \lambda_{j}$.

The first mode contains 55\% of the overall energy, with $65 \%$ of the energy represented by the first three modes ( $80 \%$ for the first 20 modes). From the fourth mode on, the energy contribution of each mode is always lower than $2 \%$ of the total energy, with only $35 \%$ of the energy being associated with the remaining 4322 modes. The first three modes will be discussed in more detail in Sects. 5.3 and 5.4.

\subsection{Spatial modes}

\subsubsection{First POD mode}

The first spatial mode shown in Fig. 12 is associated with velocity fluctuations in the separated and in the shock oscillation area. The most important variations are involving both the vertical displacement of the shear layer and the trailing edge separated area. The latter region of oscillation is well described by the v-component, and it starts at 55\% of the chord towards the trailing edge, whereas the former starts at $40 \%$ of the chord (most upstream location at which separation is occurring) and persists until downstream of the trailing edge.

The other aspect which is characterised by the first mode is the fluctuations induced by the shock movement which are observed between 30 and $50 \%$ of the chord, Fig. 12, left), in

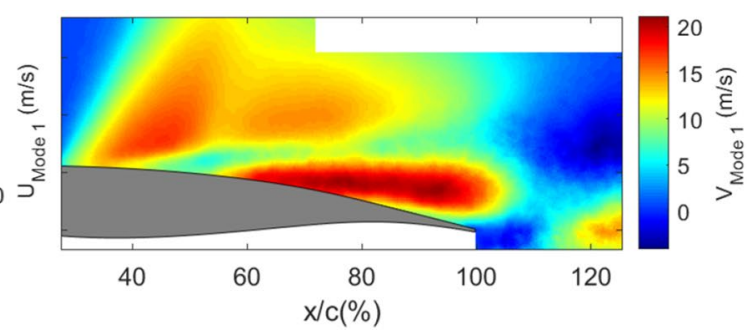

Fig. 12 First spatial POD mode for horizontal (left) and vertical (right) velocity component 

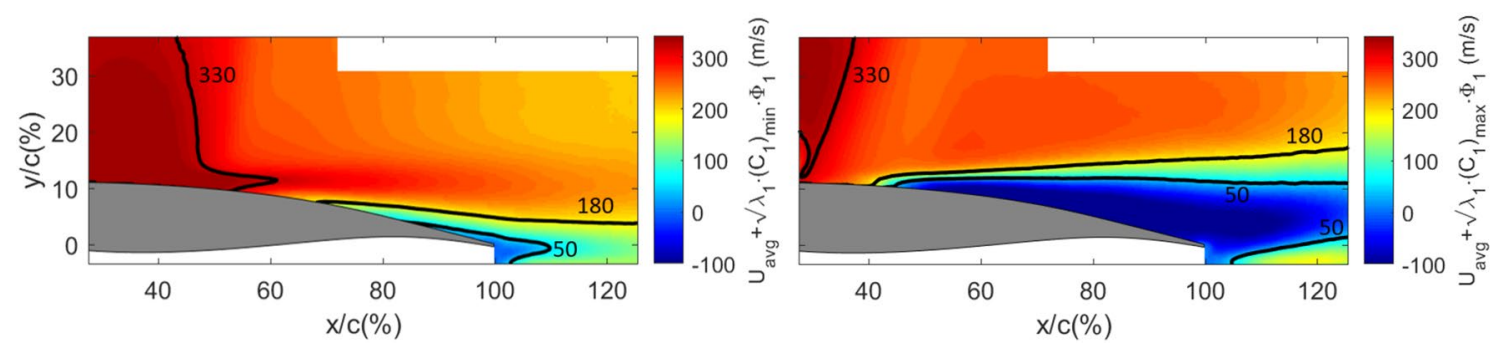

Fig. 13 First spatial mode subtracted (left) and summed (right) to the average flow field, with contour lines at 330, 180, 50 m/s
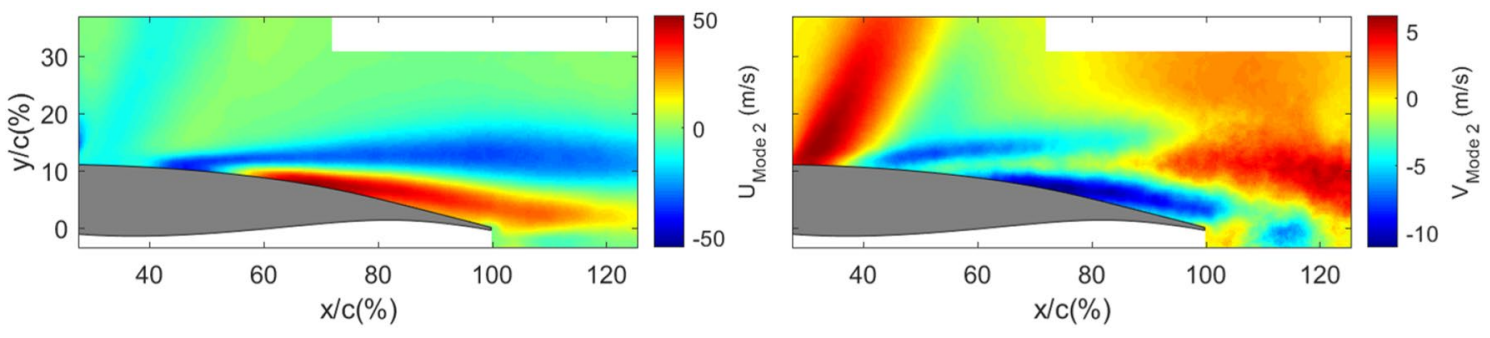

Fig. 14 Second spatial POD mode for horizontal (left) and vertical (right) velocity component
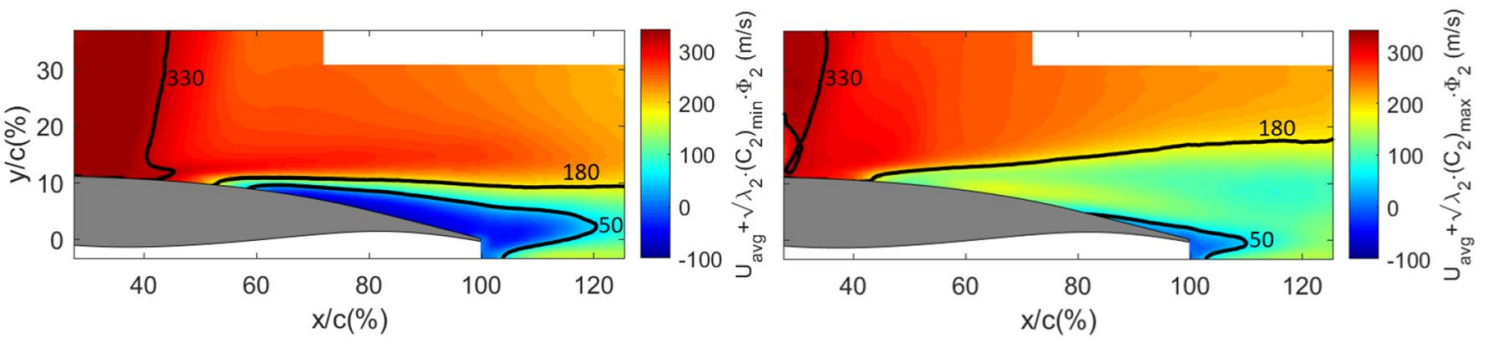

Fig. 15 Second spatial mode subtracted (left) and summed (right) to the average flow field

agreement with the phase average analysis of Sect. 4. In the presence of an oblique SW (occurring during the upstream movement), an increase in velocity is observed at the shock foot, which is described in Fig. 12 (right) by the area of positive velocity close to $40 \% \mathrm{c}$.

To better assess the contribution of the first mode to the flow dynamics, the spatial mode has been added and subtracted to the average flow field using Eq. 12. By doing so, the two extreme conditions in which the mode is in the peak (most positive time coefficient) and in the valley (most negative time coefficient) of its temporal variation are visualised. The results are discussed with visualisation of only the u-component of the velocity. The results obtained for the first mode are shown in Fig. 13, where on the left the mode is subtracted while on the right it is added. In order to better appreciate the general flow field, three contour lines have been added, corresponding to 330,180 and $50 \mathrm{~m} / \mathrm{s}$, respectively. This representation distinguishes a situation in which the separated area is wide and the shock wave oblique (right) from a situation in which the shock wave is more normal and located downstream with the separated area almost absent $(l e f t)$. It is evident that between these two extreme situations no strong variation occurs in the shear layer thickness (which is approximated by the region in between the contour lines belonging to 180 and $50 \mathrm{~m} / \mathrm{s}$ ), but only in its position.

\subsubsection{Second POD mode}

The second mode can be associated with the temporal asymmetry between the behaviour of the shear layer and of the separated area. As evident in Fig. 14, the main activity of the second mode occurs in the separated area. As shown for the u-component (Fig. 14, left), in the region where the flow is separated the mode is divided into two horizontal bands of opposite sign. This describes the behaviour of the shear layer and the separated area during the buffet cycle, with the 
possibility for the shear layer to expand when the separated area is reducing and vice versa. As a consequence, when the shear layer becomes thicker, the streamlines are deflected more upwards, while when the shear layer gets thinner, the streamlines are tilted downwards as shown by the vertical component (Fig. 14, right).

By subtracting and adding the second mode to the average flow field (Fig. 15), its contribution to the flow dynamics is visualised. In fact, when adding this mode to the average velocity field, a small separated area and a thick shear layer is obtained (right); on the other hand, when the mode is subtracted a contraction of the shear layer and an increase in the separated trailing edge area are observed (right). Thus, this mode represents the thinning and thickening of the separated shear layer. The most upstream location of the shear layer region is seen to remain basically the same (between 45 and $50 \%$ c); therefore, this mode is purely representing the variable thickness of the shear layer. In addition, a variation in the shock position is present as well, but no variation in the shock shape is observed.

\subsubsection{Third POD mode}

The third mode (Fig. 16) can be mainly associated with shock dynamics. The u-component of this mode shows important velocity variations in the region where the shock oscillates (between 30 and 50\% of the chord) and smaller fluctuations in the trailing edge area. Because of the shock oscillation, a deflection of the flow in correspondence of the SW is observed. In addition, this mode is able to account for flow separation at the shock foot (between 50 and $65 \%$ c). Both these aspects are represented by the vertical component of the third mode (Fig. 16, right).

When subtracting and adding the mode (Fig. 17), it is clear that its main contribution is to the shock oscillation area. In addition, when the shock is located more downstream (adding the mode to the average velocity field, right), a change in the structure of the shock wave in the region close to the surface is observed and with it an upstream elongation of the separated trailing edge area gets closer to the shock wave position. A small increase in the extent of the shear layer is observed as well.

\subsubsection{Reduced-order model based on the first three modes}

As mentioned in Sect. 5.2, higher modes are not further considered here, in view of their low energy contribution, with $65 \%$ of the overall energy already being represented by the first three modes. This choice is further justified in Sect. 5.4.3.

In the following section, the main buffet flow features will be further analysed using the first three modes only, which according to the previous results capture the essential components of the buffet mechanism:

- Mode 1: Breathing of the separated region in correspondence to the movement of the shock;

- Mode 2: Expansion and contraction of the shear layer;

- Mode 3: Higher-order representation of the shock movement and separated region breathing.

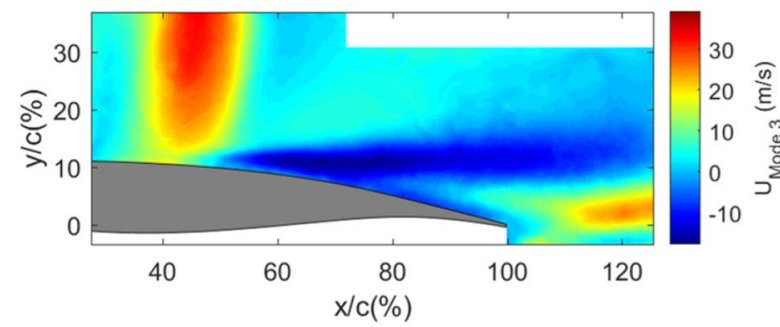

Fig. 16 Third spatial POD mode for horizontal (left) and vertical (right) velocity component
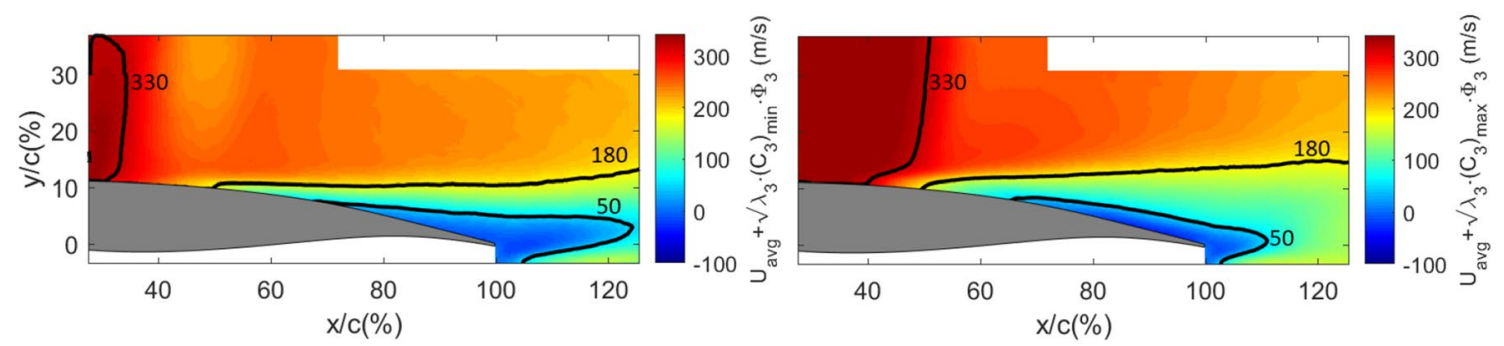

Fig. 17 Third spatial mode subtracted (left) and summed (right) to the average flow field 
When comparing this results with literature, it is worth to stress that the obtained ranking of the modes depends highly on the choice of the FOV and on the physical quantity described (in this study velocity fields).

Poplingher et al. (2019) and Ren et al. (2020) showed leading POD and DMD modes with main activity in the shockwave oscillation range and with limited or no activity in the separated area. Differently in Feldhusen-Hoffmann et al. (2021) and in Szubert et al. (2015), leading spatial modes with variations in both the shockwave oscillation range and the separated area are shown, as in this study. However, it should be noted that in Poplingher et al. (2019) and Ren et al. (2020) the spatial modes are related to the pressure field, while in Feldhusen-Hoffmann et al. (2021) and in Szubert et al. (2015) to the streamwise velocity component. This difference in visualisation of the spatial mode is confirmed in Giannelis et al. (2020). In this last study, simultaneous pressure and the streamwise velocity DMD modes are shown, confirming the presence of activity in the separated area for the latter only.

\subsection{Time coefficients}

The temporal contribution of the modes to the flow field is addressed by an analysis of the time coefficients.

In Fig. 18, the variation in the time coefficients $C_{1}, C_{2}$ and $C_{3}$, connected to the first three modes, is shown, together with the shock position (black curve), for a short time interval covering approximately four buffet cycles to illustrate their typical behaviour. Note that with the main buffet frequency being $160 \mathrm{~Hz}$, the cycle period corresponds to $6.25 \mathrm{~ms}$. The temporal coefficients clearly reflect the periodic nature of the flow field. When comparing their behaviour with the shock wave position (black line), a phase shift is observed, with none of the modes being exactly in phase with the shock position. This observation is quantified in Fig. 19, where the cross-correlation between the three time coefficients and the shock position is shown. A

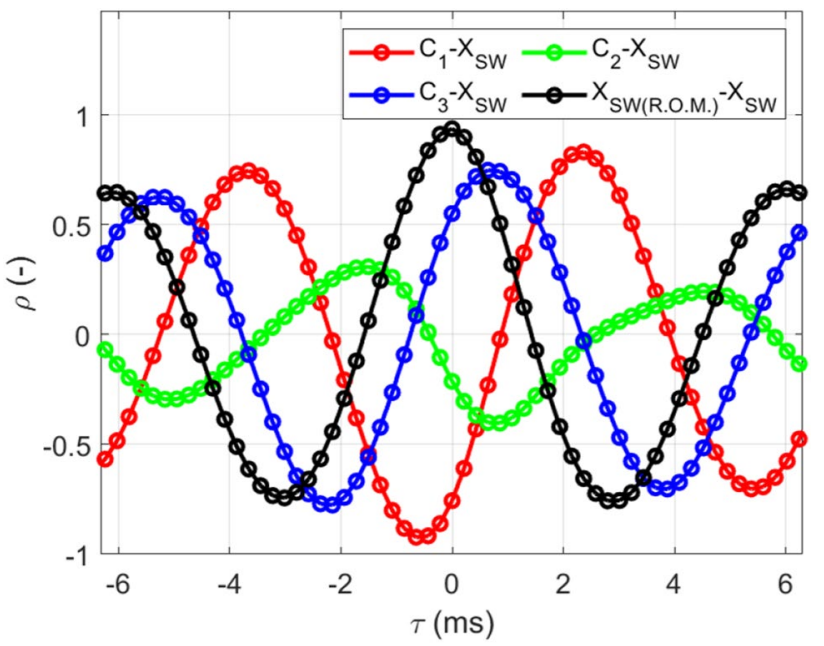

Fig. 19 Cross-correlation between shock position and first three time coefficients

high correlation value is present for both the first and the third-mode time coefficient, albeit with a phase delay, with $\left(\rho_{1, S W}\right)_{\max }=0.95$ and $\left(\rho_{3, S W}\right)_{\max }=0.8$, whereas a much weaker correlation is present for the second mode, where $\left(\rho_{2, S W}\right)_{\max }=0.3$. The fourth (black) curve represents the correlation for the shock position that is obtained with the reduced-order model based on these first three modes. The high correlation value and zero phase shift confirm that the combination of these three modes indeed provides an accurate and complete reconstruction of the buffet cycle.

To analyse the value of the time coefficients in a more quantitative way, their distribution is shown in Fig. 20 (left) by means of a pdf $\left(p\left(C_{n}\right)\right)$. It is highlighted that the $C_{2}$ coefficient has an almost symmetrical distribution with respect to the zero, while $C_{3}$ and in particular $C_{1}$ are highly skewed. This aspect is further discussed in Sect. 5.4.1.

By means of a cross-correlation procedure, the temporal relation between different modes is described, as shown in Fig. 20 (right). By virtue of the orthogonality of the
Fig. 18 Time behaviour of $C_{1}$, $C_{2}, C_{3}$ and shock position

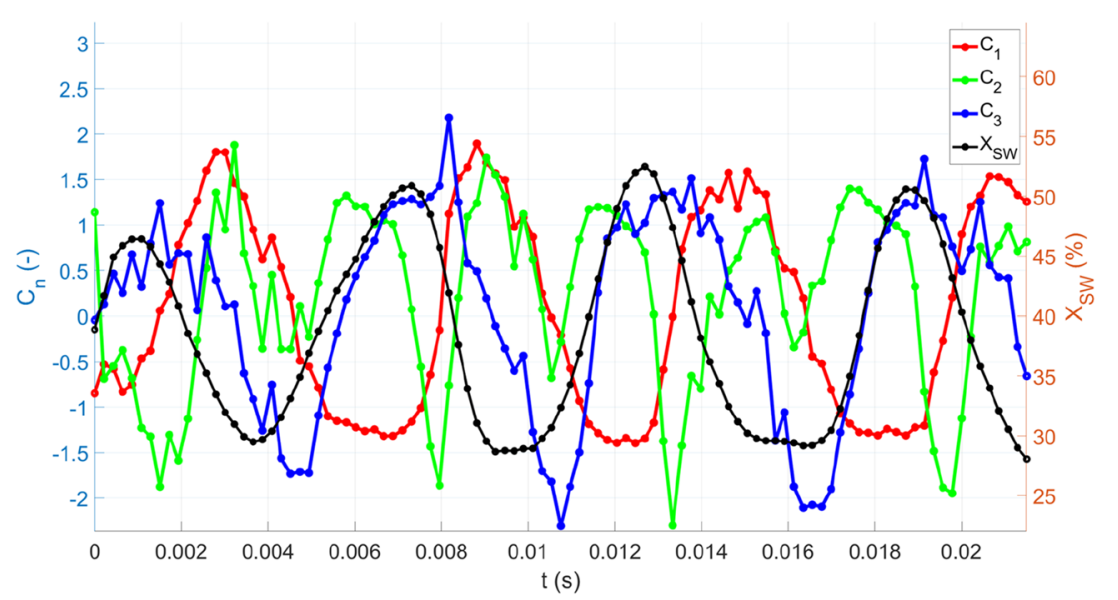



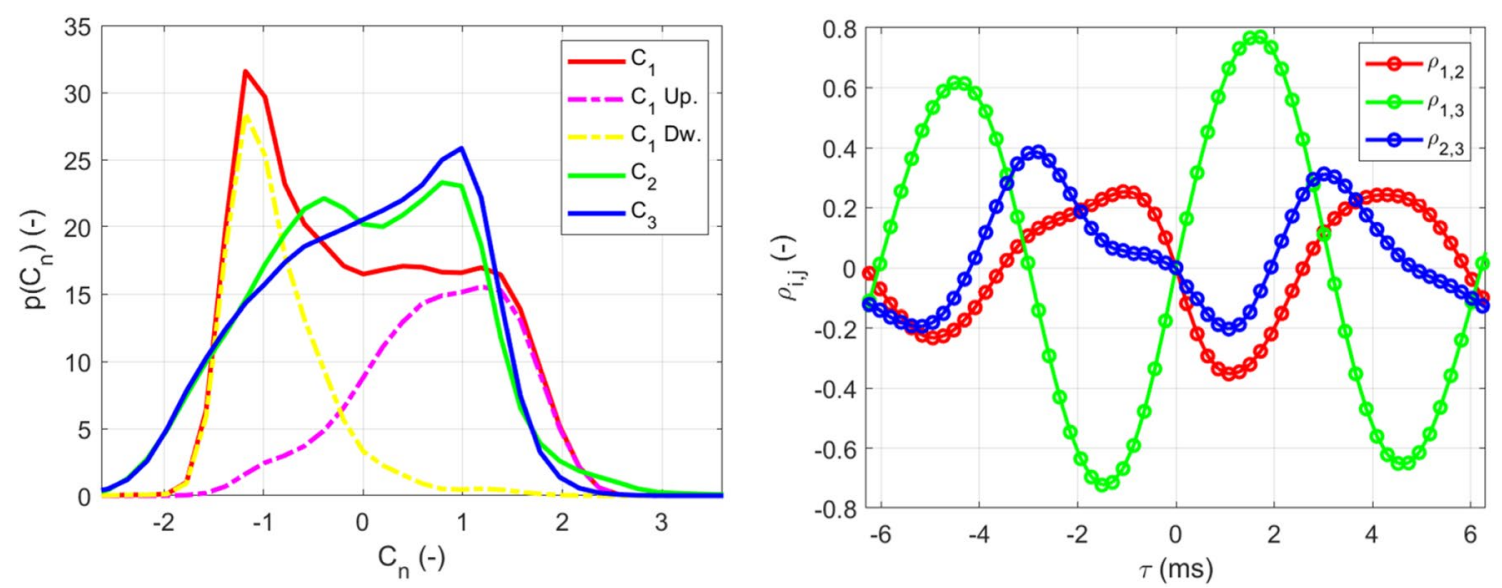

Fig. 20 Pdf of time coefficients (left) and cross-correlation between first three time coefficients (right)

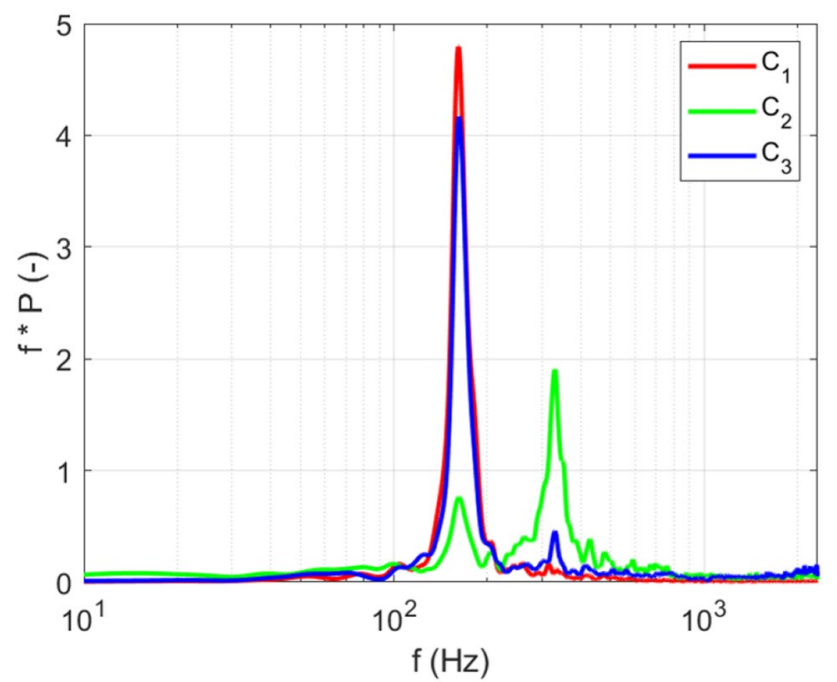

Fig. 21 PSD associated with the first three modes

POD decomposition, the cross-correlation between different modes is zero for zero lag, while the time delay $(\tau)$ for which the maximum of the cross-correlation coefficient is reached represents the phase lag between the modes. From the results, it is clear that only the first and third modes are highly correlated, $\left(\rho_{1,3}\right)_{\max }=0.75$ with a lag of $\tau=1.72 \mathrm{~ms}$ (which is approximately a quarter period). Cross-correlation values lower than 0.4 are instead obtained for both $\rho_{1,2}$ and $\rho_{2,3}$, because the dominant frequency of mode 2 lies around twice the buffet frequency (see Fig. 18 and the subsequent frequency analysis).

The spectral content of each mode is evaluated by means of the pre-multiplied PSD of the time coefficients, using again the Welch method, and the results are presented in Fig. 21. The first and third modes show a dominant peak at $160 \mathrm{~Hz}$ with negligible contributions at higher frequencies (compare Fig. 5, right). The second mode, despite having a local maximum at $160 \mathrm{~Hz}$, has the highest peak at $320 \mathrm{~Hz}$. Therefore, all the most relevant frequencies shown by the spectrogram in Fig. 10 are well represented by the spectral contribution of the first three modes.

From this analysis, it is evident that the first three modes are all strongly associated with the buffet phenomenon, albeit with different aspects of the cycle with all having the main peak at the same or at a multiple of the buffet frequency.

In the following sections, each of these first three modes, and in particular their time coefficients, will be scrutinised more in detail to better understand their physical interpretation.

\subsubsection{First-mode time coefficient}

From Fig. 18, the time coefficient $C_{1}$ appears to be related to the shock oscillation direction (velocity), being positive when the shock moves upstream and negative during the downstream phase. In particular, the $C_{1}$ coefficient reaches its local maximum or minimum in regions where the SW has the highest and lowest relative velocity with respect to the flow. This description is consistent with the behaviour of the separated area, which is reducing during the downstream travel and increasing when the shock moves upstream. When looking at one oscillation period, it is observed that the values of $C_{1}$ are almost constant during the downstream phase while varying much more during the upstream phase, once again highlighting the asymmetry between these two phases of the cycle. This observation is confirmed by the probability density functions of the first-mode time coefficients shown in Fig. 20 (left). A clear peak in the neighbourhood of the most negative value $\left(C_{1}=-1.25\right)$ is obtained, confirming that the downstream movement of the shock wave occurs in a more repeatable way; on the other hand, a broader distribution of the coefficient occurs for the positive values, which 
corresponds to the upstream motion of the shock. These conclusions are confirmed by the probability density functions of the $C_{1}$ coefficient obtained for only the upstream $\left(C_{1} \mathrm{Up}\right.$.) and only the downstream movement $\left(C_{1}\right.$ Dw.), which are shown in the same plot.

The fact that this mode is mainly connected with the separated area is further shown in Fig. 22 which shows the correlation between the $C_{1}$ coefficient, the extent of the separated area $(S)$ and the shockwave position. The extent of the separated area is evaluated by computing for each PIV snapshot the percentage of vectors which in the trailing edge area (in a region extending from 60 to $100 \% \mathrm{c}$ and with a height of $25 \%$ c) have a velocity lower than $50 \mathrm{~m} / \mathrm{s}$.

The orange line displays the correlation between the separated area and the $C_{1}$ coefficient, showing that the two signals are in phase and highly correlated, with a value of correlation close to unity occurring for $\tau=0$. On the other hand, when looking at the correlation between the separated area and the shockwave position, a negative correlation is observed, indicating that increasing values of the SW position (SW located more downstream) are associated with decreasing values of separated area. The delay between the SW signal and the separated area signal is equal to $\tau=0.645 \mathrm{~ms}$ (three snapshots) indicating that the signals are shifted by one phase of the buffet cycle (as defined in Fig. 7). The delay between the two signals is such that the shock after reaching its most downstream position starts its upstream travel. As a consequence, the SW increases the relative velocity with respect to the flow and therefore its strength, causing an increase in the extent of the separated area as well. This is consistent with what has been observed for the schlieren snapshots (Fig. 4). As expected, a very similar behaviour is obtained for the correlation between the $C_{1}$ coefficient and the shockwave position.

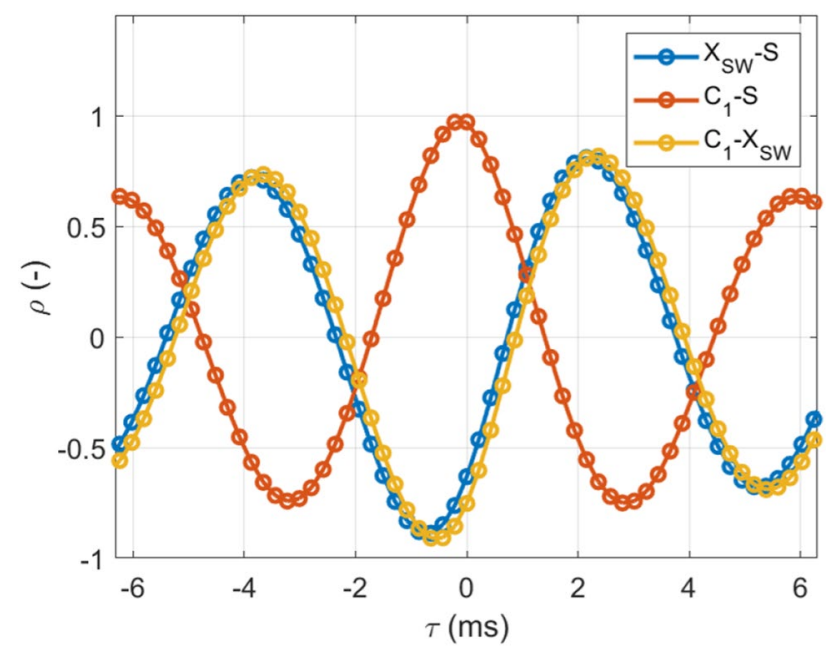

Fig. 22 Cross-correlation between first-mode time coefficient $\left(C_{1}\right)$, separated area $(\mathrm{S})$ and shockwave position $\left(X_{S W}\right)$
The pulsating behaviour of the separated area is clearly shown in literature, as for example in Jacquin et al. (2009) and Grossi et al. (2014). In the latter, when the shockwave approaches its most downstream position, the separated area spans for the first time from the shock foot till the trailing edge. However, a two-dimensional quantitative evaluation of the separated area size and its correlation with the SW position signal was not carried out, differently from the current study.

\subsubsection{Second-mode time coefficient}

The behaviour of the second time coefficient $\left(C_{2}\right)$ is instead more complex. The values of $C_{2}$ are mainly positive, with negative peaks appearing shortly after a most upstream or most downstream shock position has been reached, with the most negative value being attained in the neighbourhood of the most downstream position of the SW (see Fig. 18).

Remembering that the second mode was found to be associated with variation in the thickness of the shear layer (see Fig. 15), the time behaviour of the shear layer extent has been cross-correlated with the $C_{2}$ time behaviour. The extent of the shear layer $(S L)$ in time has been evaluated from the instantaneous images by determining the percentage of vectors with a horizontal velocity between and 50 and $220 \mathrm{~m} / \mathrm{s}$. The results obtained are shown in Fig. 23 (left) and highlight that the $C_{2}$ coefficient and the shear layer extent are perfectly in phase ( $\rho_{\max }$ for $\left.\tau=0\right)$ and correlated $(\rho(0)=0.58)$ as expected.

By evaluating the pre-multiplied PSD of the shear layer extent (Fig. 23, right), two main spectral contributions arise, the primary peak at $160 \mathrm{~Hz}$ (and therefore still associated with the basic buffet harmonic frequency) and a second harmonic at $320 \mathrm{~Hz}$, which corresponds to the frequency obtained for the $C_{2}$ coefficient in Fig. 21 (right). The results are compared with the extent of the separated area which displays a single dominant frequency at $160 \mathrm{~Hz}$ similarly to what was obtained for $C_{1}$. This result confirms that the shear layer and the separated area region have a different behaviour in time. The peak at $320 \mathrm{~Hz}$ corresponds to the fact that the shear layer is contracting just after that the SW starts its upstream travel and again after that the SW starts its downstream movement (so twice during the buffet cycle).

At the beginning of the upstream travel, the SW experiences a sharp change in inclination, which may be associated with variations in shear layer thickness.

In order to investigate such relation, the shock inclination angle $(\sigma)$ with respect to the free stream direction has been computed for all the snapshots by fitting a straight line to the shock. In Fig. 24, the simultaneous value of the shock inclination (a value lower than $90^{\circ}$ indicates backward leaning shock) and of the $C_{2}$ coefficient is shown for a limited time interval. Despite the two signals not 

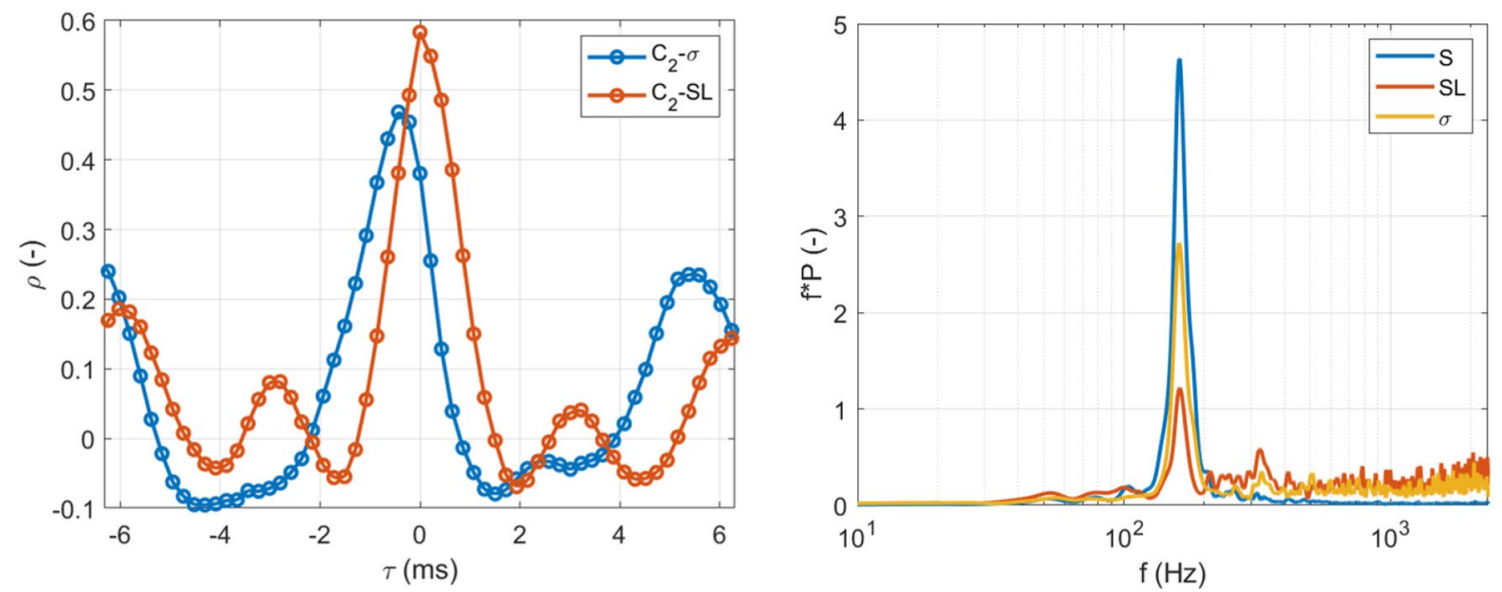

Fig. 23 On the left cross-correlation between $C_{2}$, SW inclination angle $(\sigma)$ and shear layer extent (SL). On the right PSD of the extent of the shear layer, separated area and SW inclination

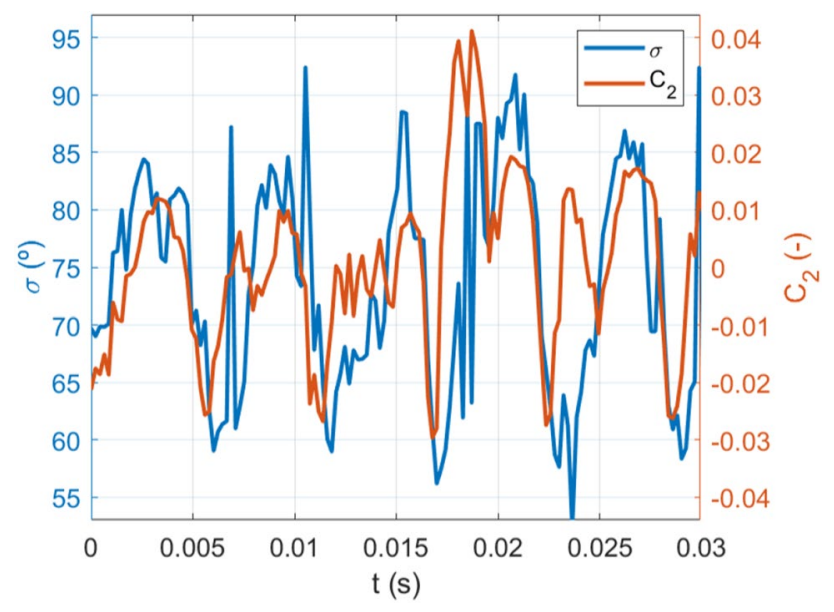

Fig. 24 Time behaviour of $C_{2}$ and of SW inclination $(\sigma)$

being in perfect agreement, there is a good correlation between the moment where the $C_{2}$ coefficient has its local minimum and the moment in which the inclination of the SW changes abruptly. This is represented in a more quantitative way by their correlation, see Fig. 23 (left) where a maximum value of $\rho=0.48$ is obtained for $\tau=-0.215 \mathrm{~ms}$ (corresponding to one snapshot interval), suggesting that the reduction in the extent of the shear layer (and increase in the separated area) causes the sudden change of the inclination of the SW and not vice versa. Therefore, this mode could account for the rapid change in the extent of the separated area which is present in the first part of the upstream travel. The spectral content of the SW inclination signal is shown in Fig. 23 (right). There is a main contribution at $160 \mathrm{~Hz}$ but also a secondary one at $320 \mathrm{~Hz}$, confirming once again the link with the flow phenomena captured by the second mode.

\subsubsection{Third-mode time coefficient}

The third mode seems to be the mode which more closely represents the shock behaviour, as can be seen in the spatial mode analysis (Fig. 17), and with $C_{3}$ decreasing from a positive to a negative value during the upstream movement and increasing in the downstream travel (Fig. 18), albeit with a small time lag with respect to the SW position (blue line in Fig. 19).

As previously discussed, the first mode is perfectly in phase with the extent of the separated area and therefore out of phase with the shock position by $0.65 \mathrm{~ms}$ (see Fig. 22); thus, this mode is not sufficient to correctly reconstruct the SW position and the extent of the separated area. In addition, as discussed in Sect. 5.4, by looking at the correlation between $C_{3}$ and the shock position it is clear that they are not in phase as well, with $C_{3}$ anticipated of $0.65 \mathrm{~ms}$ (about one phase of the buffet cycle) with respect to the shock position. Therefore, by using the first, second and third modes, a reconstructed image, which is perfectly in phase with the instantaneous snapshot in terms of SW position, is obtained, as already anticipated in Sect. 5.4. To show this, a reconstruction of the velocity field with the first three modes has been computed using Eq. 12. The shock position was subsequently detected for the resulting reconstructed images. A cross-correlation has been performed between this value of the (reconstructed) SW position $X_{S W(R . O . M)}$ and the one obtained from the instantaneous images (real SW position, $X_{S W}$ ), with the results plotted in Fig. 19. As it is clear (black line), there is no lag between the two signals, confirming the importance of the third mode to properly reconstruct the shock dynamics which, with the first mode only, would not be possible. In addition, as anticipated previously a reliable spatial and temporal reconstruction of the shear layer and separated area is achieved by using the first and the second 
mode. This justifies the use of only the first three modes to reconstruct the dynamics of the shock, shear layer and separated region.

\section{UTWs and vortex dynamics}

The first three modes accurately describe the large scale structures that characterise the buffet cycle, but they are not able to describe further aspects of the buffet oscillation, notably the propagation of the UTWs and the vortex shedding responsible for the production of the UTWs. In the literature, there is no study in which the UTW propagation is directly extracted by modal analysis. This is caused by the relatively low perturbation of velocity induced by the UTWs on the mean flow. On the other hand, the vortex shedding mechanism is successfully described by modal decomposition in many studies, such as Szubert et al. (2015) and Feldhusen-Hoffmann et al. (2021). In the current study, the vortex shedding mode shape is only observed from the 12 th mode onwards. However, none of these modes present a predominant frequency peak (or spectral bump), as should be expected for the particular phenomenon described. In fact, because of the limited acquisition frequency of the dataset, the unsteadiness associated with the vortex shedding mechanism, and the modulation with the buffet cycle, the relative dynamics is expected to be described by a multitude of modes. Therefore, none of these modes could be singularly associated with the vortex shedding phenomenon, which takes place during the buffet cycle.
Following this discussion, the POD modes are instead employed to analyse the small-scale structures in the velocity field in the form of a high pass filter, rather than by directly analysing individual modes. To achieve this, the small scale details present in the velocity field are highlighted by subtracting a reduced-order model (ROM) based on a subset of POD modes from the instantaneous velocity field. The ROM corresponds to a low-order reconstruction of the velocity field, according to Eq. 12. The ROM is based on the first 11 modes (which captures $75 \%$ of the total fluctuating kinetic energy), without including any mode with a vortex shedding shape. The additional modes included in the ROM (modes 4 to 11 ) are higher-order modes refining the SW oscillation area, the separated area and the shear layer.

\subsection{UTW}

In Fig. 25, the reconstruction of an instantaneous velocity field (u-component) based on the first 11 modes (left) is compared to the corresponding instantaneous PIV snapshot (right). A good agreement regarding the overall flow organisation is observed, notably in terms of the extent of the separated area and of the supersonic region. As expected, the small structures that are present in the separated area are lost in the reconstruction although the size of the separated area and the shock position are well conserved, while the change in velocity across the shockwave is slightly more gradual than in the real case.

By subtracting the reduced-order model reconstruction from the instantaneous velocity field, the resulting
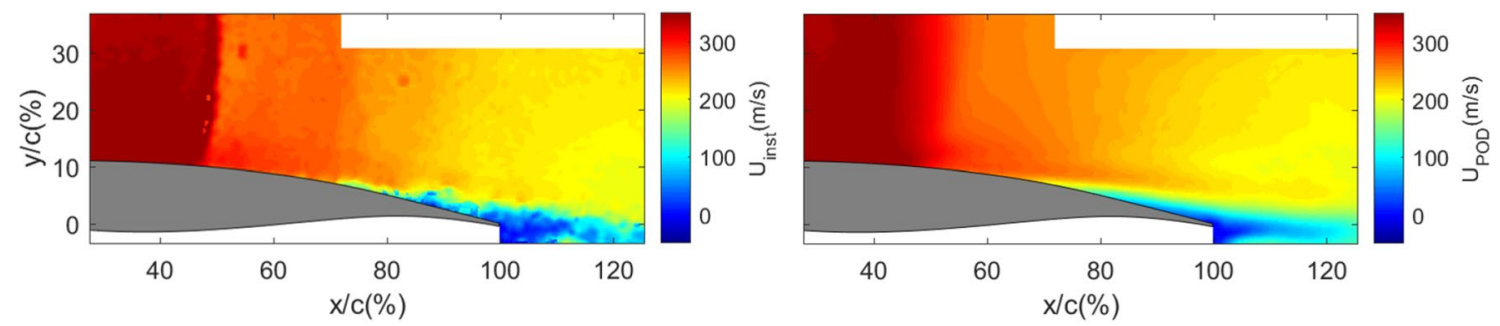

Fig. 25 Comparison between reconstructed horizontal velocity field (right) and corresponding instantaneous image (left)
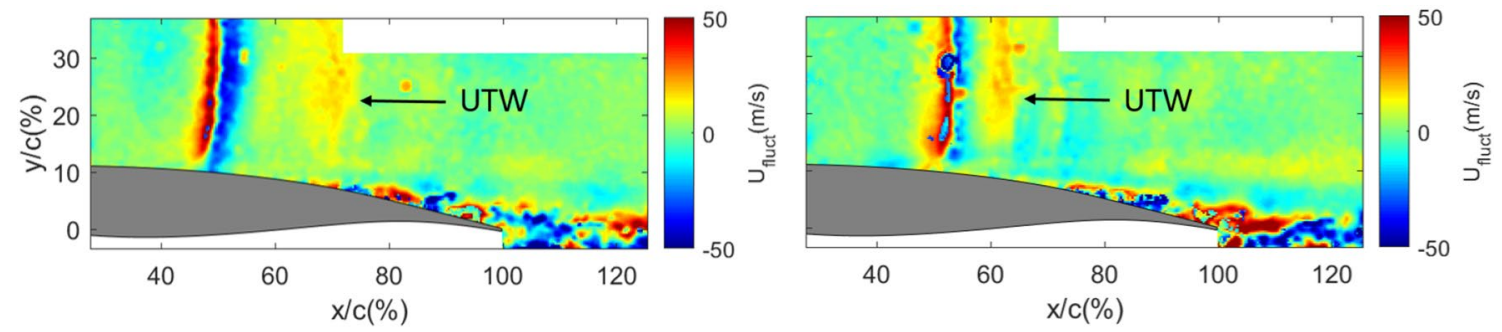

Fig. 26 Horizontal fluctuation velocity for two consecutive time steps 
fluctuating velocity field for the horizontal component is obtained (Fig. 26, left).

In the shock area, two parallel vertical structures can be observed, one red and one blue, which in modal analysis are typically connected with the use of a subrange of the total number of modes (see also the higher-order modes in Szubert et al. 2015 and Poplingher et al. 2019). From this image, vortices produced in the separated trailing edge area as well as the occurrence of UTWs (yellow vertical structures) are identified. The fluctuating velocity field for the consecutive time step is shown in the image on the right of Fig. 26.

In Fig. 26, the UTW is visualised as the marked yellow region that in the first image appears at around $70 \%$ of the chord, whereas in the following time step it has been moved forward to a position at around $60 \%$ of the chord. From the displacement of the UTW between two consecutive time steps, its propagation velocity can be evaluated yielding approximately $46 \mathrm{~m} / \mathrm{s}$ for the wave shown in (Fig. 26). This velocity is in perfect agreement with $U_{l o c}-a_{l o c}=45 \mathrm{~m} / \mathrm{s}$, which is the speed of sound with respect to the flow at that location. The agreement between this estimation and the actual experimental observation confirms that the observed structure is an upstream propagating pressure wave. However, as the UTWs are relatively weak flow features, for which the associated variation in flow velocity is small, the detection of the UTWs is not possible in every PIV snapshot. As a consequence, an evaluation of the shedding frequency of the UTWs is not possible. In order to have a more quantitative analysis regarding these waves, one may refer to Hartmann et al. (2013), where an average velocity of $80 \mathrm{~m} / \mathrm{s}$ is reported for the UTWs, or to the study by D'Aguanno et al. (2019) in which a clear detection of these waves is achieved by means of the BOS technique. The latter work shows that their velocities range between 40 and $100 \mathrm{~m} / \mathrm{s}$, moving always at the speed of sound with respect to the local flow velocity. In D'Aguanno et al. (2019), a shedding frequency of the UTWs of approximately $2000 \mathrm{~Hz}$ is obtained, in good agreement in terms of Strouhal number $(S t \approx 1)$ with the value reported by Hartmann et al. (2013).

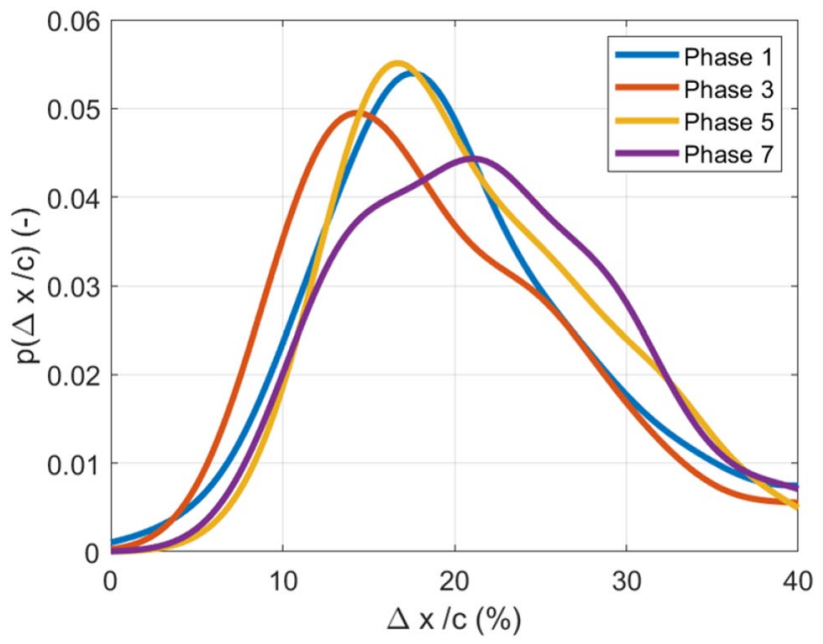

Fig. 28 Pdf of vortex wavelength per phase, obtained subtracting the first 11 POD modes

\subsection{Shear layer vortices}

When applied to the vertical velocity component, the subtraction of the reconstructed and instantaneous velocity fields allows to detect the presence of vortices in the separated area as shown in Fig. 27 (left), where the vortices are represented by areas of alternating velocity sign. By computing the autocorrelation in the rectangular region indicated in Fig. 27 (left), the distance between two consecutive vortices can be determined, therefore obtaining their wavelength.

Figure 27 (right) gives the autocorrelation map corresponding to the instantaneous velocity field visualised on the left. Relative to the origin, there is a main negative peak at $0.07 \mathrm{c}$ and a secondary positive peak at approximately double the distance (distance between consecutive vortices). However, because of the variation in time of the extent of the separated area, the autocorrelation images are such that it is not always possible to unambiguously detect a second positive peak because of the lack, for some snapshots, of consecutive vortices.

For this reason, after extending this procedure to all of the instantaneous images, the distance between the central peak and first negative peak has been evaluated as the measure of
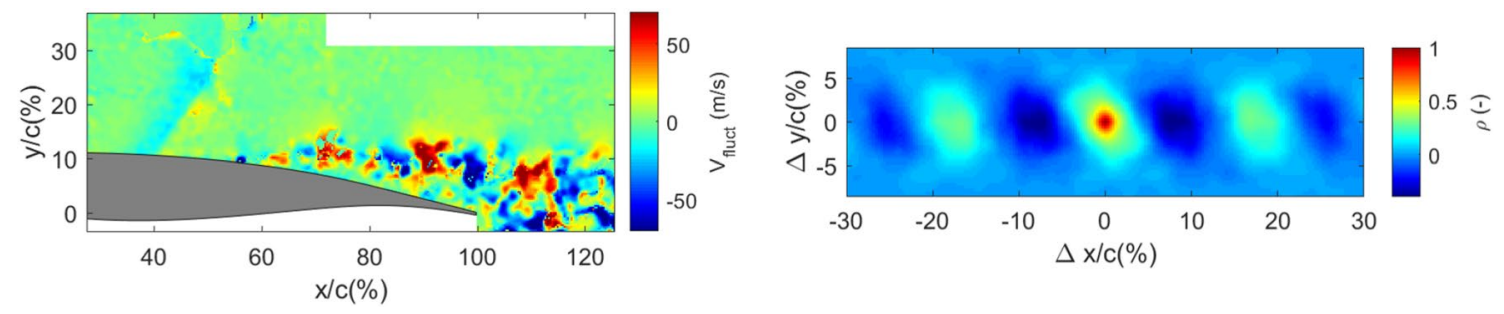

Fig. 27 Autocorrelation (right) of POD subtracted vertical velocity field (on the left) 
the mean vortex separation distance. Considering that the generation of the downstream travelling waves (DTWs) is linked with complete vortex-shedding cycles, its wavelength $(\Delta x)$ has been computed as double the vortex distance identified before. The probability density function of the wavelength $p(\Delta x / c)$ has been evaluated separately for each phase (Fig. 28) and is discussed here for the phases 1, 3, 5, 7. For different phases, the most frequent wavelength is ranging between 15 and $21 \% \mathrm{c}$. The distribution is narrower for phase 1 and 5 , when the $\mathrm{SW}$ is in the most extreme positions of the buffet cycle, while is wider when the SW is moving (higher uncertainty). It is also observed that the wavelength is shorter during the downstream travel.

As a first estimate, the propagation velocity of the vortices is computed as the average of the horizontal velocity in the shear layer region (included in the area where the crosscorrelation analysis was carried out, see Fig. 27). An average velocity in the range of $100-140 \mathrm{~m} / \mathrm{s}$ is obtained, which is in good agreement with the approximations of the convection velocity present in the literature for similar applications (see Smits and Dussauge 2006). From these data, the shedding frequency of the DTWs is estimated as:

$f_{D T W s}=\frac{u_{D T W s}}{\Delta x_{D T W s}}$

which yields a shedding frequency of between 5000 and $8000 \mathrm{~Hz}(\mathrm{St}=2.2-3.5)$ which is much higher than the frequency obtained for the UTWs by D'Aguanno et al. (2019) and Hartmann et al. (2013). It should be noted that a more accurate evaluation of the shedding frequency can be obtained from a direct temporal cross-correlation of the fluctuating velocity field, which, however, requires a much higher acquisition frequency than presently available.

\section{Discussion and conclusions}

The present investigation has addressed the dynamics of transonic buffet of the OAT15A airfoil (under fully developed conditions, at $M a=0.7$ and $\alpha=3.5^{\circ}$ ), using highspeed schlieren and PIV, supported with a phase-averaged description as well as modal analysis by means of snapshot POD.

The POD analysis shows how the buffet cycle displays an asymmetric behaviour, with the phase of buffet in which the shock moves downstream being very different from the phase when it moves upstream. In the upstream phase, the shock is moving faster with an opposite velocity with respect to the flow which causes an increase in shock strength, in combination with the occurrence of an enlarged separated area. On the other hand, the downstream phase is characterised by a weaker shock strength and smaller separated area. The increase in strength of the SW during the upstream movement is documented also in Iovnovich and Raveh (2012) as a result of the simultaneous change in orientation of the SW and increase in the Mach number ahead of the SW (because of the shock motion velocity).

As revealed by the cross-correlation between the shockwave and the separated area, a phase lead of the shock position with respect to the separated area size is observed (Fig. 22). That means that the SW starts its upstream movement before the (relatively sudden) increase in the separated area occurs, suggesting that the increase in the separated area size is a consequence and not a cause of the SW upstream movement. Therefore, the upstream travel cannot be justified without considering the presence of additional structures, confirming the role played by the UTWs.

The upstream movement of the shock is sustained by the increasing pressure downstream of the shock and by the interaction with the UTWs, while the downstream movement is characterised by a decreasing pressure downstream of the shock position associated with the reduction in the size of the separated area. This results in the shock being faster in the upstream phase (as shown in Fig. 8) while moving in a slower and steadier way during the downstream travel (see Fig. 20, left). The reduction in velocity could be justified by the fact that during the upstream shock movement, the UTWs have the same direction of propagation as the SW (differently from the downstream phase). In fact when the UTWs travel upstream, behind the UTWs the pressure is slightly higher, requiring the SW to become weaker and hence move towards a region with a lower Mach number; the simultaneous growth of the separated area (and the related increase in pressure) could explain the difference in velocity between the two phases.

An estimation of the characteristic velocity of the UTWs was accomplished, confirming that these waves behave as pressure waves, moving at the speed of sound relative to the flow. These results are in good agreement with those reported in literature, where furthermore a shedding frequency in the order of $2000 \mathrm{~Hz}$ for the UTWs was reported (D'Aguanno et al. 2019; Hartmann et al. 2013 in terms of Strouhal number). On the other hand, when analysing the vortex shedding in the separated area, a frequency in the range of $5000-8000 \mathrm{~Hz}$ is obtained in the present investigation. As this value is discordant with the frequency predicted for the UTWs, it suggests that these features are not correlated. The frequency range of the vortex structures observed is in good agreement in terms of Strouhal number ( $\mathrm{St}=$ 2.2-3.5) with the frequency obtained in Szubert et al. (2015) $(\mathrm{St}=2.6)$ for the von Kármán shedding.

In this study, it has been assumed that the UTWs are produced when vortical structures convecting downstream pass over the trailing edge (i.e. from the wall bounded shear layer to a free shear layer). In view of the observed frequency 
discrepancy, this explanation suggests that the vortices created at the shock foot, which then convect into the separated region in an area detached from the airfoil, cannot be responsible for the creation of the UTWs. Therefore, there must be other structures whose passage over the trailing edge of the airfoil causes the production of pressure waves (UTWs), and so close the buffet feedback mechanism. Even though this could not be proved unambiguously in the present visualisation, due to the lack of spatial resolution of the PIV images in the area close to the surface of the airfoil, it is speculated that the structures which are causing the generation of the UTWs are produced in the separated trailing edge area (instead of the shock foot separated area). In particular, a trailing edge separated area can occur even when no separation is triggered at the shock foot. An example of this is shown in Fig. 25 (left) where an instantaneous PIV image is visualised during the downstream travel of the SW (when there is no shock foot separated area), with separated trailing edge area arising from nearly $70 \%$ of the chord. The vortices present in this area are strongly affected by the pulsation of the shock foot separated area: in more detail, when the shock foot separated area is not present, vortices of high intensity are created since high variations in velocity are taking place across a small region (high shear levels). Differently, when the separated area is increasing its dimensions, vortices of lower intensity are shed. This could explain how a phenomenon, with a characteristic frequency of $2000 \mathrm{~Hz}$, could influence buffet which occurs at $160 \mathrm{~Hz}$. Hence, the strength of the UTWs is supposed to be modulated in accordance with the buffet frequency by means of the pulsation of the separated area, which this study has demonstrated that it oscillates with the buffet frequency at $160 \mathrm{~Hz}$ (see blue line in Fig. 23, right).

Both the schlieren instantaneous images (Fig. 4) and the phase averaged PIV velocity fields (Fig. 9) showed that also the orientation of the SW changes with the variation in the separated area, with the SW appearing more oblique during its upstream movement and more normal during its downstream motion. It is also worth stressing that the shock abruptly changes its inclination at the beginning of the upstream movement. This event is associated with a simultaneous change in the size of the shear layer, which is well described by the second POD mode (see Fig. 24). This mode is also activated at the beginning of the downstream travel, but with lower relevance, emphasising the inherent asymmetry present in the flow between the upstream and the downstream movement.

Even though a complete comprehension of the buffet mechanism has yet to be achieved, POD has demonstrated to be a useful tool for analysing the fluid-dynamic phenomena that occur, revealing in particular the asymmetry in the buffet cycle, as well as the interrelation between different flow features involved (shock, shear layer, separated area).
As such, it is considered that the present finding contributes to the knowledge of the physical mechanisms involved in buffet.

Acknowledgements This work has been carried out as part of the project HOMER (Holistic Optical Metrology for Aero-Elastic Research), funded by the European Commission, Program H2020 under Grant No. 769237.

Open Access This article is licensed under a Creative Commons Attribution 4.0 International License, which permits use, sharing, adaptation, distribution and reproduction in any medium or format, as long as you give appropriate credit to the original author(s) and the source, provide a link to the Creative Commons licence, and indicate if changes were made. The images or other third party material in this article are included in the article's Creative Commons licence, unless indicated otherwise in a credit line to the material. If material is not included in the article's Creative Commons licence and your intended use is not permitted by statutory regulation or exceeds the permitted use, you will need to obtain permission directly from the copyright holder. To view a copy of this licence, visit http://creativecommons.org/licenses/by/4.0/.

\section{References}

Arányi P, Janiga G, Zähringer K, Thévenin D (2013) Analysis of different POD methods for PIV-measurements in complex unsteady flows. Int J Heat Fluid Flow 43:204-211

Benedict LH, Gould RD (1996) Towards better uncertainty estimates for turbulence statistics. Exp Fluids 22:129-136

Coleman H, Steele W (2009) Experimentation, validation, and uncertainty analysis for engineers, 3rd edn. John Wiley \& Sons

Crouch JD, Garbaruk A, Magidov D, Jacquin L (2009) Global structure of buffeting flow on transonic airfoils. In: IUTAM symposium on unsteady separated flows and their control. pp 297-306

Crouch JD, Garbaruk A, Magidov D (2007) Predicting the onset of flow unsteadiness based on global instability. J Comput Phys 224:924-940

D’Aguanno A, Schrijer FFJ, van Oudheusden BW (2019) Study of upstream travelling waves in transonic buffet. In: Proceedings of the 13th international symposium on particle image velocimetry (ISPIV) (2019) July 22-24, 2019. Munich, Germany

de Kat R, van Oudheusden BW (2012) Instantaneous planar pressure determination from PIV in turbulent flow. Exp Fluids 52:1089-1106

Deck S (2005) Numerical simulation of transonic buffet over a supercritical airfoil. AIAA J 43:1556-1566

Feldhusen-Hoffmann A, Lagemann C, Loosen S, Meysonnat P, Klaas M, Schröder W (2021) Analysis of transonic buffet using dynamic mode decomposition. Exp Fluids 62:66

Giannelis NF, Levinski O, Vio GA (2020) Origins of atypical shock buffet motions on a supercritical aerofoil. Aerosp Sci Technol 107:106304

Giannelis NF, Vio GA, Levinski O (2017) A review of recent developments in the understanding of transonic shock buffet. Prog Aerosp Sci 92:39-84

Grossi F, Braza M, Hoarau Y (2014) Prediction of transonic buffet by delayed detached-eddy simulation. AIAA J 52:2300-2312

Hall KC, Dowell EH, Thomas JP (2000) Proper orthogonal decomposition technique for transonic unsteady aerodynamic flows. AIAA J 38:1853-1862 
Hartmann A, Feldhusen A, Schröder W (2013) On the interaction of shock waves and sound waves in transonic buffet flow. Phys Fluids 25(2):026101

Hilton WF, Fowler RG (1952) Photographs of shock wave movement. Technical Report No. 2692. HM Stationery Office

Humble RA (2009) Unsteady flow organization of a shock wave/boundary layer interaction. Ph.D. thesis. Delft University of Technology. ISBN: 978-90-597-2295-8

Iovnovich M, Raveh D (2012) Reynolds-averaged Navier-Stokes study of the shock-buffet instability mechanism. AIAA J 50:880-890

Jacquin L, Molton P, Deck S, Maury B, Soulevant D (2009) Experimental study of shock oscillation over a transonic supercritical profile. AIAA J 47:1985-1994

Lee BHK (1990) Transonic buffet on a supercritical aerofoil. Aeronaut J 94:143-152

Masini L, Timme S, Peace A (2019) Reynolds number effects on wing shock buffet unsteadiness. In: AIAA 2019-2820, AIAA Aviation (2019) Forum, 17-21 June 2019. Dallas, Texas

Melling A (1997) Tracer particles and seeding for particle image velocimetry. Exp Fluids 8:1406-1416

Ohmichi Y, Ishida T, Hashimoto A (2018) Modal decomposition analysis of three-dimensional transonic buffet phenomenon on a swept wing. AIAA J 56:1-13

Poplingher L, Raveh DE, Dowell EH (2019) Modal analysis of transonic shock buffet on 2D airfoil. AIAA J 57:2851-2866

Ragni D, Schrijer FFJ, van Oudheusden BW, Scarano F (2011) Particle tracer response across shocks measured by PIV. Exp Fluids 50:53-64

Ren K, Chen Y, Gao C, Zhang W (2020) Adaptive control of transonic buffet flows over an airfoil. Phys Fluids 32:096106
Schrijer FFJ, Solana Perez R, van Oudheusden BW (2018) Investigation of transonic buffet using high speed PIV. In: Proceedings of the 5th international conference on experimental fluid mechanics (ICEFM (2018) July 2-4, 2018. Munich, Germany

Schrijer FFJ, Scarano F (2008) Effect of predictor-corrector filtering on the stability and spatial resolution of iterative PIV interrogation. Exp Fluids 45:927-941

Sciacchitano A, Wieneke B (2016) PIV uncertainty propagation. Meas Sci Technol 27:084006

Sirovich L (1987) Turbulence and the dynamics of coherent structures. I-coherent structures. II-symmetries and transformations. IIIdynamics and scaling. Q Appl Math 45:561-590

Smits AJ and Dussauge JP (2006) Turbulent shear layers in supersonic flow. Springer Science \& Business Media

Sun Z (2014) Micro ramps in supersonic turbulent boundary layers: an experimental and numerical study. Ph.D. thesis. Delft University of Technology. 978-94-619-1924-3

Szubert D, Grossi F, Jimenez Garcia A, Hoarau Y, Hunt J, Braza M (2015) Shock-vortex shear-layer interaction in the transonic flow around a supercritical airfoil at high Reynolds number in buffet conditions. J Fluids Struct 55:276-302

Welch P (1967) The use of fast Fourier transform for the estimation of power spectra: a method based on time averaging over short, modified periodograms. IEEE Trans Audio Electroacoust 15:70-73

Publisher's Note Springer Nature remains neutral with regard to jurisdictional claims in published maps and institutional affiliations. 